\title{
Dynamics of supercooled liquids: density fluctuations and Mode Coupling Theory
}

Preprint submitted to Journal of Physics: Condensed Matter (October 2001).

\author{
E. Zaccarelli ${ }^{1}$, G. Foffi ${ }^{1}$, P. De Gregorio ${ }^{1}$, F. Sciortino ${ }^{2}$, \\ P. Tartaglia ${ }^{2}$ and K. A. Dawson ${ }^{1}$ \\ ${ }^{1}$ Irish Centre for Colloid Science and Biomaterials, Department of Chemistry, \\ University College Dublin, Belfield, Dublin 4, Ireland \\ 2 Dipartimento di Fisica, Università di Roma La Sapienza, Istituto Nazionale di \\ Fisica della Materia, and INFM Center for Statistical Mechanics and Complexity, \\ Piazzale Aldo Moro 2, 00185 Roma, Italy
}

\begin{abstract}
We write equations of motion for density variables that are equivalent to Newtons equations. We then propose a set of trial equations parameterised by two unknown functions to describe the exact equations. These are chosen to best fit the exact Newtonian equations. Following established ideas, we choose to separate these trial functions into a set representing integrable motions of density waves, and a set containing all effects of non-integrability. The density waves are found to have the dispersion of sound waves, and this ensures that the interactions between the independent waves are minimised. Furthermore, it transpires that the static structure factor is fixed by this minimum condition to be the solution of the Yvon-BornGreen (YBG) equation. The residual interactions between density waves are explicitly isolated in their Newtonian representation and expanded by choosing the dominant objects in the phase space of the system, that can be represented by a dissipative term with memory and a random noise. This provides a mapping between deterministic and stochastic dynamics. Imposing the Fluctuation-Dissipation Theorem (FDT) allows us to calculate the memory kernel. We write exactly the expression for it, following two different routes, i.e. using explicitly Newtons equations, or instead, their implicit form, that must be projected onto density pairs, as in the development of the well-established Mode Coupling Theory (MCT). We compare these two ways of proceeding, showing the necessity to enforce a new equation of constraint for the two schemes to be consistent. Thus, while in the first 'Newtonian' representation a simple gaussian approximation for the random process leads easily to the Mean Spherical Approximation (MSA) for the statics and to MCT for the dynamics of the system, in the second case higher levels of approximation are required to have a fully consistent theory.
\end{abstract}

\section{Introduction}

The field of equilibrium statistical mechanics was founded on the simple but remarkable proposition that motions generated by Newtonian or Hamiltonian mechanics have very simple statistical properties if there are sufficiently large numbers of interacting particles. Specifically, the earliest developments of statistical mechanics presupposed the so-called 
theorem of molecular chaos which assumed that all parts of phase-space are equally probable [1]. This simple idea is at the basis of the ergodic theorems, which state the equality of time and ensemble averages, the latter having an explicit representation in the Boltzmann probability distribution. Subsequent developments further strengthened the foundations of statistical mechanics by weakening the molecular chaos requirement, and the hypothesis of equal probability for all parts of phase space, while retaining the formalism of statistical mechanics. Other related developments allow to study the dynamics of many particle systems using some sort of stochastic process that mimics aspects of the chaotic motions of Newtonian mechanics. Indeed, various advances have provided a firmer basis for relating Newtonian mechanical averages to the statistical properties of stochastic averages [2, 3].

Somewhat deeper problems arise when one wants to study those systems where the parts of phase space explored over time are limited, and limited in what is perhaps no longer a simple manner. Already approaching an equilibrium phase transition, a system becomes strongly non-ergodic. Indeed, when a phase transition occurs, by the breaking of a symmetry for example, it is no longer appropriate to average over all parts of the phase space in the probability averages of Boltzmann. Instead we average only over those parts close to the lowest, most probable energy state [1]. In such simple cases the Boltzmann machinery, applied to a limited portion of phase space, can still be applied. However, when the ergodicity is lost in the system in a less well defined manner it may not be possible to find any straightforward generalisation of the Boltzmann distribution, and one is forced to reconsider the possibility to perform time averages with respect to another type of averaging. The provision of a well defined mechanism to do this is, in the arena of spin glasses, one of the substantial achievements of that field [5].

Where dealing with particles, whether they be atoms, molecules, or colloidal particles, we know at least that Newtonian dynamics, or some equivalent stochastic process, remains faithful to the physical picture, and averaging with respect to such a dynamics would be satisfactory. Examples of systems where these questions arise include structural glasses [6], the glass transition [7], and many other cases where amorphous materials such as gels [8, 9, 10], composites etc. are formed, there being no prominent state to dominate the average, and render the Boltzmann procedure acceptable. In such cases one of the few approaches able to describe the dynamics of the system is the ideal Mode Coupling Theory (MCT) [11, [12]. In fact, as we shall see, one way of viewing MCT is as an approximate method of performing Newtonian averages [13]. In this sense $\mathrm{MCT}$ is an important exemplar of a new way of thinking about calculating statistical dynamical averages, without depending on any form of ergodic hypothesis, or on any assumption that the long time probability distribution is the Boltzmann one.

In this paper, we shall present a formalism that connects Newtonian averages taken with respect to initial positions and momenta to stochastic averages taken with respect to noise distributions. This formalism has much in common with the Zwanzig-Mori formalism [2], but we here emphasise as much as possible the connection and fidelity to Newtonian averages, and the possibility to explicitly realise these averages within 
various approximations.

An important aspect of the approach lies in its transparency. This allows the possibility of an easy generalisation, for example, to consider other types of collective variables, other than simply density waves, as well as to represent non-integrable parts of motion of the system not only by linear dissipation and random noise, as we will discuss here. We will also attempt, where possible, to probe the physical meaning of the various steps, and above all the limitations of the approximations made. One interesting consequence of our approach is that ideal MCT emerges as a consistent and natural first 'mean-field' type approximation [13, 14, 15] to our theoretical framework, thereby providing an alternative route to the MCT as a theory of dynamics, consistent with the Random Phase Approximation (RPA) of statics. In this regard, we can now consider MCT to be exact in the weak coupling limit.

Thus, we begin by writing equations of motion for the density variables that are equivalent to Newtons equations. We then propose a set of trial equations, parameterised by a set of unknown functions, to describe these exact equations [16]. These parameter functions will have to be chosen to best fit the exact Newtonian equations. We choose to separate the set of trial functions in the trial equations of motion into a set representing integrable motions of density waves (the dispersions), and a set containing all the remaining effects of non-integrability. In so doing the density waves are seen to have the dispersion of sound waves, and this choice ensures that the interactions between the independent density waves is minimised. Furthermore, it transpires that the structure factor, and thereby the direct correlation function, is fixed by this minimum condition to be the solution of the Yvon-Born-Green (YBG) equation [3, 17]. As part of this process, residual interactions between the density waves are then explicitly isolated in their Newtonian representation. We therefore have an explicit Newtonian representation of the interaction force, and find a way of representing this force by expanding the trial function.

Evidently it is possible to expand these interactions in a set of functions that are best chosen to represent the dominant objects in the phase space of the system that provide for loss of the pure oscillatory motions of density waves. The key question therefore is the nature of the underlying phase space in the density representation for that state of the system we seek to describe. It is emphasised that these interaction parameters represent all the non-integrable contributions of density waves, rather than particles. Thus, the phase relations between density waves that are implied by the existence of particles, as well as the interactions of these particles and the resulting dissipation and other type of non-integrability of the underlying Newtonian system must be accommodated by the trial functions. To make these ideas more concrete we will illustrate the problem with a simple example of how the non-integrable parts of phase space can be represented by a dissipation term with memory, as well as a correlated noise to represent the more rapid fluctuations of the density caused by effects like chaotic motions. This leads to a generalised Langevin equation where the distribution of noise and the memory kernel of the dissipative term are to be determined to reflect the statistical properties of phase 
space.

It should be emphasised that, in choosing to approximate phase space statistics partly with deterministic and partly with stochastic terms, we will always naturally arrive at a stochastic process for the trial density function equation. Therefore the idea of 'best' representing Newtonian phases space motion can only be implemented in some average sense, rather than point-wise in particle position and momentum. This will mean that we must relate, and equate, general averages over the stochastic variables to those over the Newtonian space. The equality of these two averages will, at each order, define the 'goodness' of the approximation. These issues are discussed in section 5 .

Returning to the simple case where the trial forces or interactions are described by a noise and a memory kernel, to produce a conventional generalised Langevin equation it will be sufficient to apply the Fluctuation Dissipation Theorem of type two (FDT) 18] to ensure that the system can reach equilibrium in the long time limit. It is natural to ask whether the use only of these two terms in the trial forces is sufficient to represent Newtonian dynamics, and we shall discuss the question at some length in section 5.2 .

We have earlier alluded to the matter of ensuring that averages over density correlators are the same in Newtonian and stochastic spaces. Since this involves a hierarchical process, there is the practical need to truncate the program at some point. One practical possibility, outlined in section 5.3, is to ensure equality of the relevant correlators in the two spaces, and in particular to ensure that the stochastic part of the force is correlated properly with the density variables, again to some appropriate order. These considerations then lead us to a hierarchy of equilibrium correlation function equations, the first level being the YBG equation discussed above.

Following this, in section 6, we turn to the evaluation of the exact memory kernel, using the two alternative representations of the stochastic part of the force. This culminates in two exact equations (45) and (46), that represent the central results of this paper. While these equations are exact, they are not closed. Therefore, in sections 78 8 we discuss various aspects of closure strategies. The most elegant closure found to date involves assuming a Gaussian distribution for the noise, using the Newtonian represntation, and this leads us (section 7 ) directly to the known ideal MCT for supercooled liquids.

It is important to note that, while our formulation and presentation are different from earlier treatments, in many aspects there are parallels and links to previous work. Thus, in section 8 we show how to deal with the implicit representation of the force, and point out that only pairs of density variables are required for its exact specification. We also, in section 8.2, note that the Gaussian approximation can only be consistent up to first order in density variables, and also that the stochastic force cannot be consistently represented at the level of density pairs if it is gaussian distributed. Next, in section 8.3, we begin the process of seeking consistent approximations that permit the representation of the stochastic force at the level of pairs of density variables. As an example, we illustrate the Singwi-Tosi-Land-Sjolander (STLS) [19] closure is introduced as a step in this direction, but it also fails to be completely satisfactory. 
Finally in section 9, by extracting the main elements of the traditional derivation of MCT via projection operators, we will attempt to clarify the assumptions made in our derivation of MCT and in the traditional treatment [12].

\section{Density Equations of Motion}

We begin with the definition of Newton's equations,

$$
\ddot{\mathbf{r}}_{j}(t)=-\frac{1}{m} \frac{\partial \mathcal{V}}{\partial \mathbf{r}_{j}(t)}
$$

where $\mathbf{r}_{j}(t)$ are the positions of particles $j=1, N$ and $m$ is the particle mass, and we suppose that the total inter-particle potential $\mathcal{V}\left(\mathbf{r}_{1}, \ldots, \mathbf{r}_{n}\right)$ can be expressed as a sum of pair potentials,

$$
\mathcal{V}=\frac{1}{2} \sum_{m, m^{\prime}} v\left(\left|\mathbf{r}_{m}(t)-\mathbf{r}_{m^{\prime}}(t)\right|\right)
$$

The density of the $N$ particles located at positions $\mathbf{r}_{j}$ is $\rho(\mathbf{r}, t)=\sum_{j=1}^{N} \delta\left(\mathbf{r}-\mathbf{r}_{j}(t)\right)$ and its Fourier transform, which we name the density variable, is,

$$
\rho_{\mathbf{k}}(t)=\sum_{j=1}^{N} e^{i \mathbf{k} \cdot \mathbf{r}_{j}(t)}
$$

and the structure factor of the system is defined as the density-density correlation,

$$
S(k, t)=\frac{1}{N}\left\langle\rho_{-\mathbf{k}}(0) \rho_{\mathbf{k}}(t)\right\rangle
$$

which, for equal times, corresponds to the static structure factor $S_{k}=\left\langle\left|\rho_{\mathbf{k}}\right|^{2}\right\rangle$.

We, thus, write the equations of motion corresponding to these variables using the second time derivative of (3),

$$
\ddot{\rho}_{\mathbf{k}}(t)=\sum_{j} i\left(\mathbf{k} \cdot \ddot{\mathbf{r}}_{j}(t)\right) e^{i \mathbf{k} \cdot \mathbf{r}_{j}(t)}-\sum_{j}\left(\mathbf{k} \cdot \dot{\mathbf{r}}_{j}(t)\right)^{2} e^{i \mathbf{k} \cdot \mathbf{r}_{j}(t)}
$$

So far these equations are only formal equalities, but we can now eliminate the second time derivative of the positions using Newton's equations (11). To do this, we Fourier analyse equation (2) to obtain,

$$
\mathcal{V}=\frac{1}{2 V} \sum_{m, m^{\prime}} \sum_{\mathbf{k}^{\prime}} v_{k^{\prime}} e^{-i \mathbf{k}^{\prime} \cdot\left(\mathbf{r}_{m}(t)-\mathbf{r}_{m^{\prime}}(t)\right)}=\frac{1}{2 V} \sum_{\mathbf{k}^{\prime}} v_{k^{\prime}}\left(\rho_{\mathbf{k}^{\prime}}^{*} \rho_{\mathbf{k}^{\prime}}-N \delta_{k^{\prime}, 0}\right)
$$

where $\mathrm{V}$ is the total volume of the system and we have assumed that the system is isotropic, and thus, the potential depends only on the modulus of the wave-vector.

The derivative of the potential in Fourier space (6) can be written as,

$$
\frac{\partial \mathcal{V}}{\partial r_{j}(t)}=\frac{1}{2 V} \sum_{\mathbf{k}^{\prime}} v_{k^{\prime}}\left(\frac{\partial \rho_{\mathbf{k}^{\prime}}^{*}}{\partial k^{\prime}} \rho_{\mathbf{k}^{\prime}}+\rho_{\mathbf{k}^{\prime}}^{*} \frac{\partial \rho_{\mathbf{k}^{\prime}}}{\partial k^{\prime}}\right)=\frac{1}{2 V} \sum_{m} \sum_{\mathbf{k}^{\prime}} v_{k^{\prime}}\left(-i \mathbf{k}^{\prime} e^{-i \mathbf{k}^{\prime} \cdot\left(\mathbf{r}_{j}(t)-\mathbf{r}_{m}(t)\right)}+\text { c.c. }\right)
$$

so that the equations of motion for the density variables (5) now become,

$$
\begin{aligned}
\ddot{\rho}_{\mathbf{k}}(t) & =-\sum_{j}\left(\mathbf{k} \cdot \dot{\mathbf{r}}_{j}(t)\right)^{2} e^{i \mathbf{k} \cdot \mathbf{r}_{j}(t)}-\frac{1}{m V} \sum_{j, m} \sum_{\mathbf{k}^{\prime}} v_{k^{\prime}}\left(\mathbf{k} \cdot \mathbf{k}^{\prime}\right) e^{-i \mathbf{k}^{\prime} \cdot\left(\mathbf{r}_{j}(t)-\mathbf{r}_{m}(t)\right)} e^{i \mathbf{k} \cdot \mathbf{r}_{j}(t)} \\
& =-\sum_{j}\left(\mathbf{k} \cdot \dot{\mathbf{r}}_{j}(t)\right)^{2} e^{i \mathbf{k} \cdot \mathbf{r}_{j}(t)}-\frac{1}{m V} \sum_{\mathbf{k}^{\prime}} v_{k^{\prime}}\left(\mathbf{k} \cdot \mathbf{k}^{\prime}\right) \rho_{\mathbf{k}-\mathbf{k}^{\prime}}(t) \rho_{\mathbf{k}^{\prime}}(t)
\end{aligned}
$$


Note that equations (8) constitute an exact set of equations, that are equivalent to Newton's equations, but written in terms of the density variables. Clearly the first term represents the kinetic part of the motion, which is not simply expressable in terms of density variables, while the second term, originating from the interaction potential, indicates a coupling of the density pairs.

We note here that, in principle, to describe the underlying Newtonian dynamics correctly, i.e. to include sufficient information in the theory that would mimic positions and velocities of the $N$ particles, one would need to consider many fields. In particular the most general ones are usually considered the hydrodynamic variables of longitudinal and transverse currents, along with a local temperature or entropy variable [20]. Until now, mode coupling type theories have been based on longitudinal current, and therefore only the density degree of freedom, and this is the approach we will also follow. Extensions involving other fields are more complex, but, due to the transparency of the approach, it should be quite straightforward at least to write the correct equations of motion, as done in this and the next sections for the density variables.

\section{Trial equations and Residual Force between Elementary Excitations}

Equations (8), being equivalent to Newton's equations, are intractable as presently written. They must therefore be approximated in some manner. To do this we consider the possibility to isolate any simple degrees of freedom that can be easily treated, especially if the real system can be considered as some sort of perturbation around them. Natural candidates for this treatment are all integrable motions in the phase space. We will consider as fundamental collective motions for describing the system and the essential mechanisms by which its fluctuations relax the density waves.

Thus, we propose to write equations (8) in a simple 'trial' form [16]. Indeed, we consider the equations of motion, for density waves that have some interaction force between them, to be of the form,

$$
\ddot{\rho}_{\mathbf{k}}(t)+\hat{\Omega}_{\mathbf{k}} \rho_{\mathbf{k}}(t)=\hat{\mathcal{F}}_{\mathbf{k}}(t)
$$

Here, we have isolated the linear term in $\rho_{\mathbf{k}}(t)$, that represents the elementary excitations

of the system, and we have indicated by $\hat{\mathcal{F}}_{\mathbf{k}}(t)$ the interaction force between them, this being a functional of the frequencies $\hat{\Omega}_{\mathbf{k}}$ that have to be determined, as well as any other parameters to represent the forces. These elementary excitations can be interpreted as a sort of 'phonons'. Of course, these would be oscillating perfectly with the frequencies $\hat{\Omega}_{\mathbf{k}}$ if there was no interaction force between them. Thus, they represent integrable motions and we also assume that, for the purpose of describing a liquid, these are the only collective motions that need to be taken into account. The residual interaction force between these 'phonons', $\hat{\mathcal{F}}_{\mathbf{k}}(t)$, is the responsible for the deviation of the density waves from a perfect oscillatory behaviour. The smaller it is, the simpler the solutions $\rho_{\mathbf{k}}(t)$ of equations (9) will be. Generally, in a liquid, this interaction is not negligible, as it would be in a crystal. 
Now, we apply a variational principle to minimise this interaction [16, 21]. Our variational parameters are the dispersions $\hat{\Omega}_{\mathbf{k}}$. We require,

$$
\frac{\partial\left\langle\left|\hat{\mathcal{F}}_{\mathbf{k}}\right|^{2}\right\rangle_{\mathcal{N}}}{\partial \hat{\Omega}_{\mathbf{k}}}=0
$$

and thereby calculate the optimal dispersion $\Omega_{\mathbf{k}}$, that satisfies this condition and thus results in the least residual interaction between density waves.

Here the averages are performed over initial conditions of positions and velocities, $\left\{r_{n}(0), \dot{r}_{n}(0)\right\}$, or equivalently of density variables and their velocities, $\left\{\rho_{\mathbf{k}}(0), \dot{\rho}_{\mathbf{k}}(0)\right\}$, via the definition $(3)$. Therefore we have indicated them with the notation $\langle\cdots\rangle_{\mathcal{N}}$ to denote that they are Newtonian averages. We have,

$$
\frac{\partial\left\langle\left|\hat{\mathcal{F}}_{\mathbf{k}}\right|^{2}\right\rangle_{\mathcal{N}}}{\partial \hat{\Omega}_{\mathbf{k}}}=\frac{\partial\left\langle\left|\ddot{\rho}_{\mathbf{k}}(t)+\hat{\Omega}_{\mathbf{k}} \rho_{\mathbf{k}}(t)\right|^{2}\right\rangle_{\mathcal{N}}}{\partial \hat{\Omega}_{\mathbf{k}}}=\left\langle\rho_{-\mathbf{k}}\left(\ddot{\rho}_{\mathbf{k}}+\hat{\Omega}_{\mathbf{k}} \rho_{\mathbf{k}}\right)\right\rangle_{\mathcal{N}}+\left\langle\left(\ddot{\rho}_{-\mathbf{k}}+\hat{\Omega}_{\mathbf{k}} \rho_{-\mathbf{k}}\right) \rho_{\mathbf{k}}\right\rangle_{\mathcal{N}}=0
$$

so that the solution is,

$$
\Omega_{\mathbf{k}}=\frac{\left\langle\left|\dot{\rho}_{\mathbf{k}}\right|^{2}\right\rangle_{\mathcal{N}}}{\left\langle\left|\rho_{\mathbf{k}}\right|^{2}\right\rangle_{\mathcal{N}}}
$$

This is a general result. Indeed, it was shown by Zwanzig [21] to be valid for the frequencies associated with any elementary excitations in a fluid, in our case $\rho_{\mathbf{k}}(t)$. By using the definitions of density variables (3), one easily obtains,

$$
\Omega_{\mathbf{k}}=\frac{k^{2}}{\beta m S_{k}}
$$

The same result (13) can also be easily found by evaluating the short-time limit of equations (8), after multiplying them for $\rho_{-\mathbf{k}}(0)$ and performing the thermodynamical average [3, 12]. The particular derivation we have presented here is instructive in showing how the collective motions are being removed from further consideration in the dynamics of the system, as all of the attention from now on can be focused on the residual interactions between them. We can now calculate this explicitly. By adding to both sides of equations (9) the term $\Omega_{k} \rho_{\mathbf{k}}(t)$, we have an explicit form for the minimum residual force $\mathcal{F}_{\mathbf{k}}(t)=\min \hat{\mathcal{F}}_{\mathbf{k}}(t)$. Thus,

$$
\mathcal{F}_{\mathbf{k}}(t)=\Omega_{k} \rho_{\mathbf{k}}(t)-\sum_{j}\left(\mathbf{k} \cdot \dot{\mathbf{r}}_{j}(t)\right)^{2} e^{i \mathbf{k} \cdot \mathbf{r}_{j}(t)}-\frac{1}{m V} \sum_{\mathbf{k}^{\prime}} v_{k^{\prime}}\left(\mathbf{k} \cdot \mathbf{k}^{\prime}\right) \rho_{\mathbf{k}-\mathbf{k}^{\prime}}(t) \rho_{\mathbf{k}^{\prime}}(t)
$$

Clearly at some point we will have to represent this force in a simpler manner, if we are to make progress.

\section{The Yvon-Born-Green Equation}

It is of interest to note the fact that the best excitations (in the sense of minimising residual interactions between them) also implies some conditions on the interaction force. Indeed, if we refer back to the minimisation of equation (11), we can see that there an orthogonality condition is implied, i.e.

$$
\left\langle\rho_{-\mathbf{k}}(t) \mathcal{F}_{\mathbf{k}}(t)\right\rangle_{\mathcal{N}}=0
$$


This is the statement that the instantaneous force at every time must be orthogonal to the density waves themselves. This is an exact condition that should be enforced in any type of 'trial' equations of motion developed to describe Newton's equations. Thus, it must also be applied to our equations, with minimised residual force,

$$
\ddot{\rho}_{\mathbf{k}}(t)+\Omega_{\mathbf{k}} \rho_{\mathbf{k}}(t)=\mathcal{F}_{\mathbf{k}}(t)
$$

Of course, this equation can be used to determine the equilibrium correlation functions. Indeed, using the explicit expression of the residual force (14), the orthogonality condition may be written out explicitly as,

$$
\Omega_{\mathbf{k}}\left\langle\left|\rho_{\mathbf{k}}\right|^{2}\right\rangle_{\mathcal{N}}-\left\langle\sum_{j}\left(\mathbf{k} \cdot \dot{\mathbf{r}}_{j}(t)\right)^{2} e^{i \mathbf{k} \cdot \mathbf{r}_{j}(t)}\right\rangle_{\mathcal{N}}-\frac{1}{m V} \sum_{\mathbf{k}^{\prime}} v_{\mathbf{k}^{\prime}}\left(\mathbf{k} \cdot \mathbf{k}^{\prime}\right)\left\langle\rho_{-\mathbf{k}}(t) \rho_{\mathbf{k}-\mathbf{k}^{\prime}}(t) \rho_{\mathbf{k}^{\prime}}(t)\right\rangle_{\mathcal{N}}=0
$$

Now we note that the averages involved here are equilibrium averages, and as such they involve nothing more than the Boltzmann distribution. Thus, the averages of positions and momenta (or velocities) factor and can be carried out separately to yield,

$$
-\frac{k^{2}}{\beta m}\left(1-\frac{1}{S_{k}}\right) N S_{k}=\frac{1}{m V} \sum_{\mathbf{k}^{\prime}} v_{\mathbf{k}^{\prime}}\left(\mathbf{k} \cdot \mathbf{k}^{\prime}\right)\left\langle\rho_{-\mathbf{k}}(t) \rho_{\mathbf{k}-\mathbf{k}^{\prime}}(t) \rho_{\mathbf{k}^{\prime}}(t)\right\rangle_{\mathcal{N}}
$$

and defining the direct correlation function, $c_{k}=\frac{1}{n}\left(1-\frac{1}{S_{k}}\right)$, where $n=N / V$ is the number density of the system, we find,

$$
c_{k}=-\beta v_{k}-\frac{\beta}{k^{2} N^{2} S_{k}} \sum_{\mathbf{k}^{\prime} \neq \mathbf{k}} v_{\mathbf{k}^{\prime}}\left(\mathbf{k} \cdot \mathbf{k}^{\prime}\right)\left\langle\rho_{-\mathbf{k}}(t) \rho_{\mathbf{k}-\mathbf{k}^{\prime}}(t) \rho_{\mathbf{k}^{\prime}}(t)\right\rangle_{\mathcal{N}}
$$

where we have extracted from the sum the term corresponding to $\mathbf{k}=\mathbf{k}^{\prime}$ since it corresponds to simply $-\beta v_{k}$. Condition (19) constitutes a set of equations for the equilibrium fluid. It can also be shown [17] that Eq.[19] is simply the representation in Fourier space of the YBG equation. Thus, equation (19) gives a relation for the pair (static structure factor) and triplet density correlation functions in terms of the potential of the system. For example, if one could determine the static structure factor by ensuring this condition, one would have also determined the set of frequencies $\Omega_{k}$ that appear in the trial equations (16).

\section{Transition from a Deterministic to a Stochastic Dynamics}

As we commented under equation (14), it is necessary to approximate in some way the residual force to make further progress. Broadly speaking, it has always been assumed that such forces can be separated into a slowly varying and a rapidly varying part [2, 3, 22]. If we decide to represent the rapidly varying part as some type of noise, then the trial equations of motion for the density variables will become stochastic equations.

In this section we will examine the transition from a deterministic to a stochastic dynamics in its various aspects. Firstly, in subsection [5.1, we will propose a simple form for the residual minimised interactions $\mathcal{F}_{\mathbf{k}}(t)$ between density waves, basing ourselves on standard mathematical formalisms [2]. This will allow us to write the density equations of motion (8) in the form of a set of generalised Langevin equations. Then, in subsection 
5.2 we will focus on the mathematical constraints that must be imposed to our stochastic process to faithfully reproduce its 'true' Newtonian dynamics. Finally, in subsection 5.3 we propose a practical way of implementing constraints between the Newtonian and the stochastic space.

\subsection{The Physical Picture}

So far, we have written a set of trial equations (16), where all of the collective parts of the motion have been isolated and, at the same time, the interactions or effective forces between these collective motions have been minimised. The outcome is a set of collective motion frequencies (13) that are related to the structure factor, and that this structure factor is the solution of the YBG equation.

Now, the effective interactions, or trial forces, in equation (14), must be approximated to accommodate the residual physical interactions, but preserving the mathematical constraints, such as (15) discussed above, between them. A central observation is that, in the absence of the coupling terms $\mathcal{F}_{\mathbf{k}}(t)$, the collective motions would have infinite lifetimes. On the contrary, in a real system, depending on its details, we have different types of effects, tending either to cause the collective phonon-type motions to decay or to make them more long lived. Indeed, the same system, for different temperatures or densities, may exhibit such different tendencies, so that it will be important to design trial forces that are flexible enough to accommodate the particular phenomena of interest, as for example, the glass transition.

Let us first deal with the issue of particle versus wave motion. Of course, if we are able to solve Newton's equations exactly in either their familiar particle or their density representation there will be no difference. That is, we may represent all of the phenomena equally well in terms of particles, or density waves, providing every step is exact. Inevitably approximations will be introduced and then the representation that we choose will be important. For smaller wave-vectors we know that a substantial part of the motion is collective. For larger wave-vectors we expect that aspects of the independent and uncoordinated nature of the single-particle motion will be dominant. It should be noted that at long wavelengths we are dealing with waves where many particle collisions occur and where the longer density waves are locally in equilibrium. This is also reflected in the fact that we seek to model the collective oscillations using frequencies that involve the equilibrium structure factor. Therefore it is only part of the collisional spectrum that contributes to the decay of the density variables, the remainder having contributed to local equilibration of the density waves.

We now turn to the question of how the remaining effects of finite lifetimes of these waves can be described. Also, we recall that it will be important to retain at least some information on the relative phases of the density waves, so as to have an adequate description of particle, rather than wave, properties.

We assume, as usual, that the finite lifetime arises from correlated phenomena, as well as more chaotic effects. It is thus conventional to separate the residual interactions 
into two types of contribution, following the ideas of the Zwanzig-Mori formalism [2]. The first term involves a first derivative in density, so it will clearly cause a decay of the oscillating density waves. However, by using a memory kernel, we can parameterise some aspects of the history of the system. The second term is a general random noise that originates a more 'chaotic' behaviour for the system. Of course, such a separation is not a final and complete one, but it is conventional, and we will accept it for the current purposes.

By this statement we can represent our residual force (14) in the most general form using these contributions to non-integrability,

$$
\mathcal{F}_{\mathbf{k}}(t)=-\int_{0}^{t} \gamma_{\mathbf{k}}\left(t-t^{\prime}\right) \dot{\rho}_{\mathbf{k}}\left(t^{\prime}\right) d t^{\prime}+\eta_{\mathbf{k}}(t)
$$

where $\gamma_{\mathbf{k}}(t)$ is the memory function of the system and $\eta_{\mathbf{k}}(t)$ is the noise. The form (20) for the residual force must, of course, satisfy the orthogonality condition (15).

We now return briefly to the effects of having a memory kernel in equation (20). Clearly, by selecting various forms for $\gamma_{\mathbf{k}}(t)$ we are able to control the means by which dissipation is expressed in the system. Thus, short-time effects that would, for a normal liquid, lead to more rapid decay of the waves, can be incorporated. Also, more extensive collective effects involving many particles can be incorporated, in an averaged manner. Finally, again in some averaged manner, we can incorporate those aspects of phase coherences of different density waves, that are necessary to retain a minimal picture of particle motions. Indeed, potentially we are using the memory kernel to accommodate a number of different physical effects.

By this expansion, we have made a transition from a deterministic description of the system, based on equations (16) and (14), to a stochastic one, where the full equations of motions are expressed through the generalised Langevin equations,

$$
\ddot{\rho}_{\mathbf{k}}(t)+\Omega_{k} \rho_{\mathbf{k}}(t)+\int_{0}^{t} \gamma_{\mathbf{k}}\left(t-t^{\prime}\right) \dot{\rho}_{\mathbf{k}}\left(t^{\prime}\right) d t^{\prime}=\eta_{\mathbf{k}}(t)
$$

with $\Omega_{\mathbf{k}}$ given by (13) and $\gamma_{\mathbf{k}}(t)$ still to be determined. Introducing the normalised density correlator, defined as $\Phi_{k}(t)=S_{k}(t) / S_{k}$, the exact equations of motion clearly are,

$$
\ddot{\Phi}_{\mathbf{k}}(t)+\Omega_{\mathbf{k}} \Phi_{\mathbf{k}}(t)+\int_{0}^{t} \gamma_{\mathbf{k}}\left(t-t^{\prime}\right) \dot{\Phi}_{\mathbf{k}}\left(t^{\prime}\right) d t^{\prime}=0
$$

where the causality relation $\left\langle\rho_{-\mathbf{k}}(0) \eta_{\mathbf{k}}(t)\right\rangle=0$ for $t>0$ must hold.

In our system the fluctuations, represented by $\eta_{\mathbf{k}}(t)$, and the dissipations, expressed by $\gamma_{\mathbf{k}}(t)$, are not independent, and, in particular, they must satisfy the fluctuationdissipation theorem (FDT), or else no Boltzmann equilibrium distribution would be reached by the Langevin equation implied by equations (21). Thus, we have,

$$
\frac{\left\langle\eta_{-\mathbf{k}}(t) \eta_{\mathbf{k}}\left(t^{\prime}\right)\right\rangle}{\left\langle\left|\dot{\rho}_{\mathbf{k}}\right|^{2}\right\rangle}=\gamma_{\mathbf{k}}\left(t-t^{\prime}\right)
$$

This relation fixes the memory function. It can be easily shown that this relation is directly implied by our generalised Langevin equations (21), with the only assumption of 
equilibrium. To describe out-of-equilibrium dynamics, a generalization of this formalism, and consequently of FDT, is necessary, as we discussed in [23].

We now want to clarify the question of time-translational invariance of our stochastic process (21). Indeed, there is an explicit dependence on the arbitrary initial time $t=0$, at which memory effects start to be taken into account, due to the crucial choice for the residual force (201). This is done for obvious physical reasons. If we consider another Langevin process of the form,

$$
\ddot{\rho}_{\mathbf{k}}+\Omega_{k} \rho_{\mathbf{k}}(t)+\int_{-\infty}^{t} \gamma_{\mathbf{k}}\left(t-t^{\prime}\right) \dot{\rho}_{\mathbf{k}}\left(t^{\prime}\right) d t^{\prime}=\tilde{\eta}_{\mathbf{k}}(t)
$$

which is now clearly time-translational invariant, we have the following relation between our true random noise $\eta_{\mathbf{k}}(t)$ of equations (21), and this new, auxiliary, noise $\tilde{\eta}_{\mathbf{k}}(t)$ [18,

$$
\eta_{\mathbf{k}}(t)=\tilde{\eta}_{\mathbf{k}}(t)-\int_{-\infty}^{0} d t^{\prime} \gamma_{\mathbf{k}}\left(t-t^{\prime}\right) \dot{\rho}_{\mathbf{k}}\left(t^{\prime}\right)
$$

it is sufficient to ask our non-time-translational invariant noise $\eta_{\mathbf{k}}(t)$ to obey the initial conditions,

$$
\begin{array}{rl}
\left\langle\rho_{-\mathbf{k}}(0) \eta_{\mathbf{k}}(t)\right\rangle=0 & t \geq 0 \\
\left\langle\dot{\rho}_{-\mathbf{k}}(0) \eta_{\mathbf{k}}(t)\right\rangle=0 & t>0
\end{array}
$$

as well as that its autocorrelation function is the same as the one for $\tilde{\eta}_{\mathbf{k}}(t)$. Of course, we require the memory kernel,

$$
\left\langle\eta_{-\mathbf{k}}(0) \eta_{\mathbf{k}}(t)\right\rangle=\left\langle\tilde{\eta}_{-\mathbf{k}}\left(t_{1}\right) \tilde{\eta}_{\mathbf{k}}\left(t_{1}+t\right)\right\rangle=\gamma_{\mathbf{k}}(t)
$$

to ensure that the two stochastic processes (21) and (24) are completely equivalent [18]. Thus, causality is also correctly built in our equations (21).

This means that, though the random noise $\eta_{\mathbf{k}}(t)$ itself is not time-translational invariant, the whole process, described by equations (21), is. In particular, we have that the residual force $\mathcal{F}_{\mathbf{k}}(t)(20)$, which is the sum of the random noise plus the conventional memory term, starting from $t=0$, does not depend on the initial time. Indeed, it is important to clarify this aspect, as the orthogonality condition (15) between the density and the force $\mathcal{F}$ at equal times, implies,

$$
\left\langle\rho_{-\mathbf{k}}(t) \mathcal{F}_{\mathbf{k}}(t)\right\rangle=\left\langle\rho_{-\mathbf{k}}(0) \mathcal{F}_{\mathbf{k}}(0)\right\rangle=\left\langle\rho_{-\mathbf{k}}(0) \eta_{\mathbf{k}}(0)\right\rangle=0
$$

since at the initial time, we have $\mathcal{F}_{\mathbf{k}}(0)=\eta_{\mathbf{k}}(0)$. But we cannot simply say that also $\left\langle\rho_{-\mathbf{k}}(t) \eta_{\mathbf{k}}(t)\right\rangle$ is zero, because of the non-validity of the time-translational invariance for the noise $\eta_{\mathbf{k}}(t)$. In fact, for any time $t$, the orthogonality condition just implies,

$$
\left\langle\rho_{-\mathbf{k}}(t) \eta_{\mathbf{k}}(t)\right\rangle=\int_{0}^{t} \gamma_{\mathbf{k}}\left(t-t^{\prime}\right)\left\langle\rho_{-\mathbf{k}}(t) \dot{\rho}_{\mathbf{k}}\left(t^{\prime}\right)\right\rangle
$$

and, in particular, each of these terms is zero in the trivial case of a $\delta$-function memory kernel, for which, of course, the equations motion become local in time. 


\subsection{The Mathematical Constraints}

In this section we will discuss the mathematical definition of our stochastic process, and the implied constraints. This will shed some light into how good our Langevin process (21) is in reproducing the Newtonian process in equations (8). Thus, the stochastic process $\eta_{\mathbf{k}}(t)$ can be defined via the generating function,

$$
\left\langle e^{i \sum_{j} u_{\mathbf{k}_{j}}\left(t_{j}\right) \eta_{\mathbf{k}_{j}}\left(t_{j}\right)}\right\rangle_{\left\{\eta_{\mathbf{k}}\right\}}=\left\langle e^{i \sum u_{\mathbf{k}_{j}}\left(t_{j}\right)\left[\mathcal{F}_{\mathbf{k}_{j}}\left(t_{j}\right)+\int_{0}^{t_{j}} \gamma_{\mathbf{k}_{j}}\left(t_{j}-t^{\prime}\right) \dot{\rho}_{\mathbf{k}_{j}}\left(t^{\prime}\right) d t^{\prime}\right]}\right\rangle_{\left\{\mathbf{r}_{n}(0), \dot{\mathbf{r}}_{n}(0)\right\}}
$$

where the average on the lhs corresponds now to an average over the noise probability distribution. Equation (30) is, in principle, a full definition of the random process, because a stochastic process is completely defined by all its moments. Indeed, every correlation function of the noise, considered as some moment of the distribution, could be calculated by deriving it with respect to the auxiliary parameters $u_{\mathbf{k}}$ and it is thereby related directly to an average with respect to Newton's equations. These relations are only just formal, but they do place a clear set of constraints on the stochastic process that we choose to mimic Netwonian dynamics.

Besides this relation between the stochastic process and Newton's equations, there is also, via equation (21), a mapping between the density variables of the Langevin process and the noise. Indeed, Laplace transforming equations (21) and solving with respect to the density, we have,

$$
\tilde{\rho}_{\mathbf{k}}(z)=\frac{\left[-i z+\tilde{\gamma}_{\mathbf{k}}(z)\right] \rho_{\mathbf{k}}(0)+\dot{\rho}_{\mathbf{k}}(0)+\tilde{\eta}_{\mathbf{k}}(z)}{-z^{2}-i z \tilde{\gamma}_{\mathbf{k}}(z)+\Omega_{k}}
$$

where $\rho_{\mathbf{k}}(0)$ and $\dot{\rho}_{\mathbf{k}}(0)$ are the initial conditions in time of our variables, and we have used the definition of Laplace transform,

$$
\tilde{\rho}_{\mathbf{k}}(z)=\int_{0}^{\infty} d t e^{i z t} \rho_{\mathbf{k}}(t)
$$

$z$ being a complex number. Thus, now averages over density variables in the deterministic language can be replaced by averages over the noise $\eta_{\mathbf{k}}(t)$ in the stochastic one, or their equivalent density averages.

Equation (23) defines the autocorrelation function of the noise distribution, but to give a complete definition of the stochastic process we also need to define the mean value of the distribution. To do so, we can use the mapping with density variables, which implies the further condition $\left\langle\eta_{\mathbf{k}}(t)\right\rangle=0$.

Now, in principle, to create the noise distribution we should use the definition of the stochastic process, via the generating function in (30), and by taking derivatives of this, calculate the noise distribution moments satisfying this equality up to any desired accuracy. From these constraints, then, one can determine the density correlators. The level at which one truncates this program, via some closure, will be the level of approximation of the theory. Evidently, if one followed this program, all averages over any function of density variables taken in the Newtonian space could be explicitly written in terms of averages over density variables in the stochastic averages.

However, in practice we are not interested in the correlators of arbitrarily high order, nor are we interested in arbitrary functions of density variables. Indeed, what we 
still need to determine to have a complete theory is the memory kernel $\gamma_{\mathbf{k}}(t)$, which must satisfy the FDT relation (23). We will illustrate this in the following of the chapter. Nonetheless, for the present purpose, it is interesting to anticipate that the result for the memory will depend only on 2-, 3-, and 4-point density correlators for unequal times, and thus these are the only averages that need to be equal in the two spaces, Newtonian and stochastic respectively. Therefore, we should focus most attention only on these correlators.

We can write explicitly the implied equalities for 2- and 4-point correlation functions at unequal times for the noise, derived from (30), i.e.

$$
\begin{aligned}
\left\langle\eta_{-\mathbf{k}_{1}}(0) \eta_{\mathbf{k}_{2}}(t)\right\rangle_{\left\{\eta_{\mathbf{k}}\right\}}=\left\langle\mathcal{F}_{-\mathbf{k}_{1}}(0)\right. & {\left.\left[\mathcal{F}_{\mathbf{k}_{2}}(t)+\int_{0}^{t} d t^{\prime} \gamma_{\mathbf{k}_{2}}\left(t-t^{\prime}\right) \dot{\rho}_{\mathbf{k}_{2}}\left(t^{\prime}\right)\right]\right\rangle_{\left\{\mathbf{r}_{n}(0), \dot{\mathbf{r}}_{n}(0)\right\}} } \\
\left\langle\eta_{-\mathbf{k}_{1}}(0) \eta_{-\mathbf{k}_{2}}(0) \eta_{\mathbf{k}_{3}}(t) \eta_{\mathbf{k}_{4}}(t)\right\rangle_{\left\{\eta_{\mathbf{k}}\right\}}=\langle & \mathcal{F}_{-\mathbf{k}_{1}}(0) \mathcal{F}_{-\mathbf{k}_{2}}(0)\left[\mathcal{F}_{\mathbf{k}_{3}}(t)+\int_{0}^{t} d t^{\prime} \gamma_{\mathbf{k}_{3}}\left(t-t^{\prime}\right) \dot{\rho}_{\mathbf{k}_{3}}\left(t^{\prime}\right)\right] \\
& {\left.\left[\mathcal{F}_{\mathbf{k}_{4}}(t)+\int_{0}^{t} d t^{\prime} \gamma_{\mathbf{k}_{4}}\left(t-t^{\prime}\right) \dot{\rho}_{\mathbf{k}_{4}}\left(t^{\prime}\right)\right]\right\rangle_{\left\{\mathbf{r}_{n}(0), \dot{\mathbf{r}}_{n}(0)\right\}} }
\end{aligned}
$$

These equations define the stochastic averages of the correlations, or what is equivalent, the definition of the stochastic process in terms of the Newtonian dynamics, to the appropriate order. But we can further note that, using the definition of $\mathcal{F}_{\mathbf{k}}(t)$ in terms of the density variables (14) and substituting into this the expression for the densities in terms of noise (31), we obtain a hierarchy of constraints for the noise distributions. Indeed, without writing explicitly all of these contraints, it is easy to see that equation (33) implies a relationship for $\langle\eta \eta\rangle$ in terms of $\langle\eta \eta \eta\rangle$ and $\langle\eta \eta \eta \eta\rangle$. Similarly, equation (34) implies relations between $\langle\eta \eta \eta \eta\rangle$ and higher order correlations of the noise up to order $\langle\eta \eta \eta \eta \eta \eta \eta \eta\rangle$.

These constraints should be enforced for any implementation of Mori-type formalisms, with respect to the appropriate Newtonian dynamics. Then, practically, all closures of the theory involve violation of these constraints at some order of noise moments.

\subsection{Equivalent Representations of the Random Force and Practical Implementation of} Constraints

We have seen so far that the right constraints to implement for the stochastic process to represent faithfully the Newtonian process follow directly from the definition of the random noise (30). More practically, we have written explicitly the type of constraints that will be requested for our simple theory, based on the expansion (20) for the residual interactions $\mathcal{F}_{\mathbf{k}}(t)$, in (33) and (34). Now, if one correctly implemented the constraints of type (33) and (34), as well the similar ones for the triplet correlation averages that will appear in the expression for the memory kernel in the next section, the transition from Newtonian to stochastic averages would be successfully achieved for our formalism. Thus, in particular, one could consider the two sides of the exact density equations (8) to be equal when averaged with respect to any order of the density variables using the Newtonian phase space, or via the relation (31), to any order of the noise variables. 
However, the execution of this program, i.e. the exact implementation of (33) and (34), is generally difficult to pursue, and it may be of interest to ensure less extensive conditions, but ones which address the most important physical effects. The fact that the two sides of equations (8) should be equal with respect to the averaging process, to some chosen order of the density variables, at least ensures that the underlying Newtonian dynamics is reasonably reflected in the stochastic process. Thus, considering equation (20), it is clear that the random force $\eta_{\mathbf{k}}(t)$ can be explicitly written as,

$$
\eta_{\mathbf{k}}(t)=\mathcal{F}_{\mathbf{k}}(t)+\int_{0}^{t} \gamma_{\mathbf{k}}\left(t-t^{\prime}\right) \dot{\rho}_{\mathbf{k}}\left(t^{\prime}\right) d t^{\prime}
$$

but the residual force can be expressed either in its explicit form, given in (14), or in its implicit one, given by (16). Thus, we have the two equivalent representations for the noise,

$\eta_{\mathbf{k}}^{(I)}(t)=\Omega_{k} \rho_{\mathbf{k}}(t)-\sum_{j}\left(\mathbf{k} \cdot \dot{\mathbf{r}}_{j}(t)\right)^{2} e^{i \mathbf{k} \cdot \mathbf{r}_{j}(t)}-\frac{1}{m V} \sum_{\mathbf{k}^{\prime}} v_{k^{\prime}}\left(\mathbf{k} \cdot \mathbf{k}^{\prime}\right) \rho_{\mathbf{k}-\mathbf{k}^{\prime}}(t) \rho_{\mathbf{k}^{\prime}}(t)+\int_{0}^{t} d t^{\prime} \gamma_{\mathbf{k}}\left(t-t^{\prime}\right) \dot{\rho}_{\mathbf{k}}\left(t^{\prime}\right)(36)$ and,

$$
\eta_{\mathbf{k}}^{(I I)}(t)=\ddot{\rho}_{\mathbf{k}}(t)+\Omega_{k} \rho_{\mathbf{k}}(t)+\int_{0}^{t} d t^{\prime} \gamma_{\mathbf{k}}\left(t-t^{\prime}\right) \dot{\rho}_{\mathbf{k}}\left(t^{\prime}\right)
$$

For convenience of notation, we will refer from now on to eq. (36) as representation I of the random force (or explicit representation), and to eq. (37) as representation II ( or implicit representation). The requirement that the two are equal, which is to say that the decomposition of the residual force (20) is valid, is clearly equivalent to consider the equality between the two sides of equations (8), since the integral term, that we define for simplicity,

$$
\Delta_{\mathbf{k}}(t)=\int_{0}^{t} \gamma_{\mathbf{k}}\left(t-t^{\prime}\right) \dot{\rho}_{\mathbf{k}}\left(t^{\prime}\right) d t^{\prime}
$$

is equal in the two representations. Indeed, this arises from the fact that the memory function $\gamma_{\mathbf{k}}(t)$, being the correlation function of the noise following FDT (23), must also be equal in the two representations.

This will be only strictly valid if we could close exactly the hierarchy illustrated above, which clearly can also be seen as imposing constraints on the two sides of the density equations (8), or equivalently on the two representations of the noise. Since this is a far from practical program to carry on, we can try to identify those essential features of the system where consistency should be enforced, in order to render the whole approach simpler, but still satisfying the basic physical and mathematical properties.

Consider now the two representations for the random noise (36) and (37). As noted above, the two should be equal to one another with respect to any averaging process we build. A limited expression of this constraint is expressed if we ensure the validity of the equalities for any order $n$,

$$
\left\langle\left(\prod_{i=1}^{n} \rho_{-\mathbf{k}_{\mathbf{i}}}(t)\right) \eta_{\mathbf{k}}^{(I)}(t)\right\rangle=\left\langle\left(\prod_{i=1}^{n} \rho_{-\mathbf{k}_{\mathbf{i}}}(t)\right) \eta_{\mathbf{k}}^{(I I)}(t)\right\rangle
$$


This means that the two sides of equation (8) will be equal under projection onto density, density pairs, triplets and so on. It must be also noted that since these are all equal time averages they may be worked out exactly at any order and reduced to relations between the density correlation functions, and the potential. A clear example of this was given by the YBG equation (19), corresponding simply to the case $n=1$ of the general condition (39).

A second example, that will have an important part in the following discussion, is represented by the case where $n=2$, corresponding to the ensuring of the equality of the two random force representations under projection onto density pairs. Explicitly this can be written as,

$$
\left\langle\rho_{-\mathbf{p}}(t) \rho_{-\mathbf{q}}(t) \eta_{\mathbf{k}}^{(I)}(t)\right\rangle=\left\langle\rho_{-\mathbf{p}}(t) \rho_{-\mathbf{q}}(t) \eta_{\mathbf{k}}^{(I I)}(t)\right\rangle
$$

Of course, the contribution on the two sides of the integral term $\Delta_{\mathbf{k}}(t)$ (38) cancels out, because we are assuming in principle the exactness of the equality of the two representations at any level. Writing explicitly (40), we have,

$$
\begin{aligned}
& -\frac{1}{m V} \sum_{\mathbf{k}^{\prime}}\left(\mathbf{k} \cdot \mathbf{k}^{\prime}\right) v_{k^{\prime}}\left\langle\rho_{-\mathbf{p}}(t) \rho_{-\mathbf{q}}(t) \rho_{\mathbf{k}-\mathbf{k}^{\prime}}(t) \rho_{\mathbf{k}^{\prime}}(t)\right\rangle-\frac{k^{2}}{\beta m} n c_{k}\left\langle\rho_{-\mathbf{p}}(t) \rho_{-\mathbf{q}}(t) \rho_{\mathbf{k}}(t)\right\rangle \\
& =\left\langle\rho_{-\mathbf{p}}(t) \rho_{-\mathbf{q}}(t) \ddot{\rho}_{\mathbf{k}}(t)\right\rangle+\Omega_{\mathbf{k}}\left\langle\rho_{-\mathbf{p}}(t) \rho_{-\mathbf{q}}(t) \rho_{\mathbf{k}}(t)\right\rangle
\end{aligned}
$$

The terms $\left\langle\rho_{-\mathbf{p}}(t) \rho_{-\mathbf{q}}(t) \ddot{\rho}_{\mathbf{k}}(t)\right\rangle$ are easily calculated. Indeed,

$$
\left\langle\rho_{-\mathbf{p}}(t) \rho_{-\mathbf{q}}(t) \ddot{\rho}_{\mathbf{k}}(t)\right\rangle=-\left[\left\langle\dot{\rho}_{-\mathbf{p}}(t) \rho_{-\mathbf{q}}(t) \dot{\rho}_{\mathbf{k}}(t)\right\rangle+\left\langle\rho_{-\mathbf{p}}(t) \dot{\rho}_{-\mathbf{q}}(t) \dot{\rho}_{\mathbf{k}}(t)\right\rangle\right]
$$

and,

$$
\begin{aligned}
& \left\langle\dot{\rho}_{-\mathbf{p}}(t) \rho_{-\mathbf{q}}(t) \dot{\rho}_{\mathbf{k}}(t)\right\rangle=\frac{\mathbf{p} \cdot \mathbf{k}}{\beta m} \delta_{\mathbf{p}+\mathbf{q}, \mathbf{k}} N S_{q} \\
& \left\langle\rho_{-\mathbf{p}}(t) \dot{\rho}_{-\mathbf{q}}(t) \dot{\rho}_{\mathbf{k}}(t)\right\rangle=\frac{\mathbf{q} \cdot \mathbf{k}}{\beta m} \delta_{\mathbf{p}+\mathbf{q}, \mathbf{k}} N S_{p}
\end{aligned}
$$

Thus, rearranging equation (41), considering also that $\left(1-n c_{k}\right) S_{k}=1$, we finally have,

$$
\begin{aligned}
& -\frac{1}{V} \sum_{\mathbf{k}^{\prime}}\left(\mathbf{k} \cdot \mathbf{k}^{\prime}\right) v_{k^{\prime}}\left\langle\rho_{-\mathbf{p}}(t) \rho_{-(\mathbf{k}-\mathbf{p})}(t) \rho_{\mathbf{k}-\mathbf{k}^{\prime}}(t) \rho_{\mathbf{k}^{\prime}}(t)\right\rangle-\frac{k^{2}}{\beta}\left\langle\rho_{-\mathbf{p}}(t) \rho_{-(\mathbf{k}-\mathbf{p})}(t) \rho_{\mathbf{k}}(t)\right\rangle \\
& =-\frac{N}{\beta}\left\{k^{2}-\mathbf{k} \cdot \mathbf{p} n c_{p}-\mathbf{k} \cdot(\mathbf{k}-\mathbf{p}) n c_{|\mathbf{k}-\mathbf{p}|}\right\} S_{p} S_{|\mathbf{k}-\mathbf{p}|}
\end{aligned}
$$

This represents indeed a further relation involving the bare potential and the static structure factor of the system, of the same type of the YBG equation (19), but of higher order, now also involving the four density correlatione. We note that, if one consider the case $\mathbf{p}=0$ in equation (44), this simply reduces to YBG equation.

To sum up, equations (39) for any $n$ constitute a minimal set of exact equations for equilibrium correlation functions that must be satisfied if Newtons equations are to be satisfied. The first of such equations is known as the YBG equation, and has been widely studied. We have earlier shown that this consistency relationship has the effect of modelling the collective part of the particle motion in an optimal manner. The second consistency relationship has not yet been the subject of interest in liquid state theory. 
It may be expected to play a role in ensuring that leading effects of phase relations between density variables are maintained, as well as some aspects of the dissipative effects of interactions that lead to decorrelation of the density variables.

The set of equations (39) will ensure the fidelity of the Langevin process to the appropriate Newtonian dynamics on a single time slice in the history of the system. Indeed, they do not contain information about correlations between different time slices.

\section{Exact Calculation of the Memory Kernel}

Now we apply the FDT condition (23) to calculate the memory kernel. Following the definitions above, we have now two ways to proceed, by using the two equivalent representations for the random force $\eta_{\mathbf{k}}(t)$ (36),(37).

Using the explicit form (36), which contains in it the bare potential $v_{k}$, it is straightforward to write an exact expression for the memory kernel applying FDT,

$$
\begin{aligned}
& \gamma_{\mathbf{k}}^{(I)}(t)=\frac{\beta m}{N k^{2}}\left[\left(\frac{k^{2}}{\beta m}\right)^{2}\left(n^{2} c_{k}^{2}-1\right) N S_{k}(t)+\left\langle\sum_{l, m}\left(\mathbf{k} \cdot \dot{\mathbf{r}}_{l}(0)\right)^{2} e^{-i \mathbf{k} \cdot \mathbf{r}_{l}(0)}\left(\mathbf{k} \cdot \dot{\mathbf{r}}_{m}(t)\right)^{2} e^{i \mathbf{k} \cdot \mathbf{r}_{m}(t)}\right\rangle\right. \\
& +\frac{1}{(m V)^{2}} \sum_{\mathbf{k}^{\prime}} \sum_{\mathbf{k}^{\prime \prime}} v_{k^{\prime}} v_{k^{\prime \prime}}\left(\mathbf{k} \cdot \mathbf{k}^{\prime}\right)\left(-\mathbf{k} \cdot \mathbf{k}^{\prime \prime}\right)\left\langle\rho_{-\mathbf{k}-\mathbf{k}^{\prime \prime}}(0) \rho_{\mathbf{k}^{\prime \prime}}(0) \rho_{\mathbf{k}-\mathbf{k}^{\prime}}(t) \rho_{\mathbf{k}^{\prime}}(t)\right\rangle \\
& +\frac{n k^{2}}{\beta m^{2} V} c_{k}\left\{\sum_{\mathbf{k}^{\prime}} v_{k^{\prime}}\left(\mathbf{k} \cdot \mathbf{k}^{\prime}\right)\left\langle\rho_{-\mathbf{k}}(0) \rho_{\mathbf{k}-\mathbf{k}^{\prime}}(t) \rho_{\mathbf{k}^{\prime}}(t)\right\rangle+\sum_{\mathbf{k}^{\prime \prime}} v_{k^{\prime \prime}}\left(-\mathbf{k} \cdot \mathbf{k}^{\prime \prime}\right)\left\langle\rho_{-\mathbf{k}-\mathbf{k}^{\prime \prime}}(0) \rho_{\mathbf{k}^{\prime \prime}}(0) \rho_{\mathbf{k}}(t)\right\rangle\right\} \\
& \left.-\int_{0}^{t} d t^{\prime} \gamma_{\mathbf{k}}\left(t-t^{\prime}\right)\left\{\frac{k^{2}}{\beta m} n N c_{k} \dot{S}_{k}\left(t^{\prime}\right)+\frac{1}{m V} \sum_{\mathbf{k}^{\prime}} v_{k^{\prime}}\left(-\mathbf{k} \cdot \mathbf{k}^{\prime}\right)\left\langle\rho_{-\mathbf{k}-\mathbf{k}^{\prime}}(0) \rho_{\mathbf{k}^{\prime}}(0) \dot{\rho}_{\mathbf{k}}\left(t^{\prime}\right)\right\rangle\right\}\right] .
\end{aligned}
$$

Thus, to explicitly evaluate the memory kernel, it is necessary to introduce some kind of approximations to evaluate the multiple density correlations appearing in (45).

On the contrary, when using the implicit representation (II) for the random force (37), the formally exact expression for the memory kernel, would be,

$$
\begin{aligned}
\gamma_{\mathbf{k}}^{(I I)}(t) & =\frac{\beta m}{N k^{2}}\left\{\left\langle\ddot{\rho}_{-\mathbf{k}}(0) \ddot{\rho}_{\mathbf{k}}(t)\right\rangle+\Omega_{k}\left[\left\langle\ddot{\rho}_{-\mathbf{k}}(0) \rho_{\mathbf{k}}(t)\right\rangle+\left\langle\rho_{-\mathbf{k}}(0) \ddot{\rho}_{\mathbf{k}}(t)\right\rangle\right]\right. \\
& \left.+\left(\Omega_{k}\right)^{2}\left\langle\rho_{-\mathbf{k}}(0) \rho_{\mathbf{k}}(t)\right\rangle+\int_{0}^{t} d t^{\prime} \gamma_{\mathbf{k}}\left(t-t^{\prime}\right)\left\langle\left(\ddot{\rho}_{-\mathbf{k}}(0)+\Omega_{k} \rho_{-\mathbf{k}}(0)\right) \dot{\rho}_{\mathbf{k}}\left(t^{\prime}\right)\right\rangle\right\}
\end{aligned}
$$

This contains, for example, correlations at unequal times of the second derivatives of densities which are not known, even in an approximate form. Thus, to pursue this second path, we must find a more suitable form for the random force to calculate its time correlation function. This is precisely the route that has been used in the original derivation of the mode coupling theory (MCT) [12]. Clearly this second possibility, despite the presence of these intractable objects, does not contain explicitly the bare potential. Of course, in an exact expression of the theory, we should have,

$$
\gamma_{\mathbf{k}}^{(I)}(t)=\gamma_{\mathbf{k}}^{(I I)}(t)
$$

but we caution that different types of approximations made on the two expressions for the memory kernel may lead to inconsistent results. 


\section{Representation I: Random Phase Approximation and Mode Coupling Theory}

We now proceed to perform the simplest approximation to multiple density correlations, to solve the YBG equation (19) and at the same time calculate the memory function (45) in representation I. To do so, we consider the simplest case of a Gaussian random process $\eta_{\mathbf{k}}(t)$. This implies, for example, that the fourth moment of the force can be decomposed in terms of second moments, as for example,

$$
\begin{aligned}
& \left\langle\eta_{-\mathbf{k}}\left(t_{1}\right) \eta_{-\mathbf{k}}\left(t_{2}\right) \eta_{\mathbf{k}}\left(t_{3}\right) \eta_{\mathbf{k}}\left(t_{4}\right)\right\rangle=\left\langle\eta_{-\mathbf{k}}\left(t_{1}\right) \eta_{-\mathbf{k}}\left(t_{2}\right)\right\rangle\left\langle\eta_{\mathbf{k}}\left(t_{3}\right) \eta_{\mathbf{k}}\left(t_{4}\right)\right\rangle \\
& +\left\langle\eta_{-\mathbf{k}}\left(t_{1}\right) \eta_{\mathbf{k}}\left(t_{3}\right)\right\rangle\left\langle\eta_{-\mathbf{k}}\left(t_{2}\right) \eta_{\mathbf{k}}\left(t_{4}\right)\right\rangle+\left\langle\eta_{-\mathbf{k}}\left(t_{1}\right) \eta_{\mathbf{k}}\left(t_{4}\right)\right\rangle\left\langle\eta_{-\mathbf{k}}\left(t_{2}\right) \eta_{\mathbf{k}}\left(t_{3}\right)\right\rangle
\end{aligned}
$$

Since we know that density variables are linear functions of the noise from (31), also densities are then Gaussian variables. This means, for example, that the triplet correlation function appearing in the YBG equation (19) can be decomposed as,

$$
\begin{aligned}
& \left\langle\rho_{-\mathbf{k}}(t) \rho_{\mathbf{k}-\mathbf{k}^{\prime}}(t) \rho_{\mathbf{k}^{\prime}}(t)\right\rangle=\left\langle\rho_{-\mathbf{k}}(t)\right\rangle\left\langle\rho_{\mathbf{k}-\mathbf{k}^{\prime}}(t) \rho_{\mathbf{k}^{\prime}}(t)\right\rangle+ \\
& \left\langle\rho_{\mathbf{k}-\mathbf{k}^{\prime}}(t)\right\rangle\left\langle\rho_{-\mathbf{k}}(t) \rho_{\mathbf{k}^{\prime}}(t)\right\rangle+\left\langle\rho_{\mathbf{k}^{\prime}}(t)\right\rangle\left\langle\rho_{-\mathbf{k}}(t) \rho_{\mathbf{k}-\mathbf{k}^{\prime}}(t)\right\rangle
\end{aligned}
$$

and by using the general result,

$$
\left\langle\rho_{-\mathbf{p}}(t) \rho_{\mathbf{q}}(t)\right\rangle=N \delta_{\mathbf{p}, \mathbf{q}}\left[S(q)+N \delta_{\mathbf{q}, 0}\right]
$$

we have,

$$
\left\langle\rho_{-\mathbf{k}}(t) \rho_{\mathbf{k}-\mathbf{k}^{\prime}}(t) \rho_{\mathbf{k}^{\prime}}(t)\right\rangle=N \delta_{\mathbf{k}, 0}\left[S\left(k^{\prime}\right)+N \delta_{\mathbf{k}^{\prime}, 0}\right]+N \delta_{\mathbf{k}, \mathbf{k}^{\prime}}\left[S(k)+N \delta_{\mathbf{k}, 0}\right]+N \delta_{\mathbf{k}^{\prime}, 0}\left[S(k)+N \delta_{\mathbf{k}, 0}\right](51)
$$

The result of inserting (51) into (19) is that only terms where $\mathbf{k}=\mathbf{k}^{\prime}$ give a finite contribution in the sum on the right hand side, but this term has been already isolated from the rest of the sum in (19). Thus, this corresponds to a Random Phase Approximation (RPA) of the YBG equation. Therefore, we have that the structure factor is determined directly from the potential, via the relation,

$$
c_{\mathrm{k}}=-\beta v_{\mathrm{k}}
$$

Now, consistent with this approximation, we can also calculate the density averages for unequal times in (45), as for example,

$$
\begin{aligned}
& \left\langle\rho_{-\mathbf{k}-\mathbf{k}^{\prime}}(0) \rho_{\mathbf{k}^{\prime}}(0) \rho_{\mathbf{k}-\mathbf{k}^{\prime \prime}}(t) \rho_{\mathbf{k}^{\prime \prime}}(t)\right\rangle=\left\langle\rho_{-\mathbf{k}-\mathbf{k}^{\prime}}(0) \rho_{\mathbf{k}^{\prime}}(0)\right\rangle\left\langle\rho_{\mathbf{k}-\mathbf{k}^{\prime \prime}}(t) \rho_{\mathbf{k}^{\prime \prime}}(t)\right\rangle \\
& +\left\langle\rho_{-\mathbf{k}-\mathbf{k}^{\prime}}(0) \rho_{\mathbf{k}-\mathbf{k}^{\prime \prime}}(t)\right\rangle\left\langle\rho_{\mathbf{k}^{\prime}}(0) \rho_{\mathbf{k}^{\prime \prime}}(t)\right\rangle+\left\langle\rho_{-\mathbf{k}-\mathbf{k}^{\prime}}(0) \rho_{\mathbf{k}^{\prime \prime}}(t)\right\rangle\left\langle\rho_{\mathbf{k}^{\prime}}(0) \rho_{\mathbf{k}-\mathbf{k}^{\prime \prime}}(t)\right\rangle
\end{aligned}
$$

Also, to complete the calculation of the memory kernel, there is a second minor approximation that we need to introduce to evaluate the second term in the right hand side of (45). Thus, we perform the average over the velocities, even if now we are not dealing with equal time averages, and so neglecting the correlations in the single particle kinetic energy,

$$
\left\langle\sum_{l} \sum_{m}\left(\mathbf{k} \cdot \dot{\mathbf{r}}_{l}(0)\right)^{2} e^{-i \mathbf{k} \cdot \mathbf{r}_{l}(0)}\left(\mathbf{k} \cdot \dot{\mathbf{r}}_{m}(t)\right)^{2} e^{i \mathbf{k} \cdot \mathbf{r}_{m}(t)}\right\rangle \approx \frac{k^{4}}{\beta^{2} m^{2}} N S_{k}(t)
$$


Thus, the Gaussian nature of the random process, and approximation (54), allow to rewrite (45) as,

$$
\begin{aligned}
\gamma_{\mathbf{k}}(t) & =\frac{n \beta}{m V k^{2}} \sum_{\mathbf{k}^{\prime} \neq \mathbf{k}}\left\{v_{k^{\prime}}^{2}\left(\mathbf{k} \cdot \mathbf{k}^{\prime}\right)^{2}+v_{k^{\prime}} v_{k-k^{\prime}}\left(\mathbf{k} \cdot \mathbf{k}^{\prime}\right)\left(\mathbf{k} \cdot\left(\mathbf{k}-\mathbf{k}^{\prime}\right)\right)\right\} S_{\left|\mathbf{k}-\mathbf{k}^{\prime}\right|}(t) S_{k^{\prime}}(t) \\
& +\frac{k^{2} n^{2}}{\beta m}\left(c_{k}+\beta v_{k}\right)^{2} S_{k}(t)-n\left(c_{k}+\beta v_{k}\right) \int_{0}^{t} d t^{\prime} \gamma_{\mathbf{k}}\left(t-t^{\prime}\right) \partial_{t^{\prime}} S_{k}\left(t^{\prime}\right)
\end{aligned}
$$

At this point, we have to impose the YBG condition, consistent with Gaussian averages, that is the RPA condition (52), and this leads precisely to the well-known expression given by the Mode Coupling Theory (MCT) for the memory kernel,

$$
\gamma_{\mathbf{k}}(t) \stackrel{M C T}{=} \frac{n}{\beta m k^{2} V} \sum_{\mathbf{k}^{\prime} \neq \mathbf{k}}\left\{\left(\mathbf{k} \cdot \mathbf{k}^{\prime}\right)^{2} c_{\mathbf{k}^{\prime}}^{2}+\left(\mathbf{k} \cdot \mathbf{k}^{\prime}\right)\left(\mathbf{k} \cdot\left(\mathbf{k}-\mathbf{k}^{\prime}\right)\right) c_{\mathbf{k}^{\prime}} c_{\mathbf{k}-\mathbf{k}^{\prime}}\right\} S_{\left|\mathbf{k}-\mathbf{k}^{\prime}\right|}(t) S_{k^{\prime}}(t) .
$$

It is interesting to note that in the Gaussian approximation, by substituting the bare potential with the direct correlation function, the contributions to the memory kernel given by the integral term $\int_{0}^{t} \gamma_{\mathbf{k}}\left(t-t^{\prime}\right) \dot{\rho}_{\mathbf{k}}\left(t^{\prime}\right)$, present in the noise are zero. If this were not the case, the expression for the memory kernel would not be explicit any more, but it would be instead a self-consistent relation. As it will be shown later, this will be also valid if one calculates consistently in a Gaussian approximation the memory kernel in representation II, projecting onto density pairs, but leaving aside the integral term. On the contrary, in the original derivation of MCT [12], using representation II, the approximations made are not all consistent with each other, and in that derivation, this term was a priori neglected, as explained in detail in section 9 .

By introducing a factor $1 / 2$ in (56), and calling $\mathbf{k}^{\prime}=\mathbf{p}$ and $\mathbf{k}-\mathbf{k}^{\prime}=\mathbf{q}$, we can rewrite the memory kernel in the conventional MCT form, i.e.

$$
\gamma_{\mathbf{k}}(t) \stackrel{M C T}{=} \frac{n}{2 \beta m V} \sum_{\substack{\mathbf{q}+\mathbf{p}=\mathbf{k} \\ \mathbf{q} \neq 0}}\left|\left(\mathbf{q} c_{\mathbf{q}}+\mathbf{p} c_{\mathbf{p}}\right) \cdot e^{L}(\mathbf{k})\right|^{2} S_{p} S_{q} \Phi_{p}(t) \Phi_{q}(t)
$$

where $\Phi_{k}(t)$ is the normalised density correlator, for which the equations of motion are now clearly,

$$
\ddot{\Phi}_{\mathbf{k}}(t)+\Omega_{\mathbf{k}} \Phi_{\mathbf{k}}(t)+\int_{0}^{t} \gamma_{\mathbf{k}}^{M C T}\left(t-t^{\prime}\right) \dot{\Phi}_{\mathbf{k}}\left(t^{\prime}\right) d t^{\prime}=0
$$

\section{Projection onto Density Pairs}

As we have discussed earlier, the formal exact expression of the memory kernel in representation II (46) does not constitute a good starting point for making approximations and developing a simple theory, because of the presence of the second time derivatives of densities. A way to overcome this problem is to project the random force (37) onto the density pairs subspace, as it was done in the original derivation of MCT [12]. We, thus, leave unchanged the integral term $\Delta_{\mathbf{k}}(t)$ (38), which appears in the expression of the random force (35), and in particular in (37), and project the remaining part, which is clearly equal to the minimum residual force $\mathcal{F}_{\mathbf{k}}(t)$ onto the density pairs 
subspace. This choice follows naturally from the the YBG condition, which states that the residual force is orthogonal to the density at every time.

For convenience, we define a reduced random force $\tilde{\eta}_{\mathbf{k}}(t)$ that is simply the random force from which we have subtracted the term $\Delta_{\mathbf{k}}(t)$ (38), such as,

$$
\tilde{\eta}_{\mathbf{k}}(t)=\eta_{\mathbf{k}}(t)-\Delta_{\mathbf{k}}(t)
$$

To be consistent, this reduced random force coincides with the residual force $\mathcal{F}_{\mathbf{k}}(t)$, but we prefer to use this notation at this stage to underline the fact that we are talking of a stochastic process, even if mapped onto a deterministic one. As for the pure random force, also $\tilde{\eta}_{\mathbf{k}}(t)$ has of course two representations. Now, following [12], we can rewrite the random force in representation II as,

$$
\eta_{\mathbf{k}}^{(I I)}(t)=\sum_{l, m, p, s}\left\langle\rho_{-\mathbf{l}}(t) \rho_{-\mathbf{m}}(t) \tilde{\eta}_{\mathbf{k}}(t)^{(I I)}\right\rangle g(p, s, l, m) \rho_{\mathbf{p}}(t) \rho_{\mathbf{s}}(t)+\Delta_{\mathbf{k}}(t)
$$

The coefficients of the expansion $\left\langle\rho_{-\mathbf{l}}(t) \rho_{-\mathbf{m}}(t) \tilde{\eta}_{\mathbf{k}}^{(I I)}(t)\right\rangle$ can be easily calculated for the first two terms in the expression of the force (37). As we will show later, in the conventional MCT derivation, the integral term is simply neglected in the calculation of the memory kernel. The unknown matrix $g(p, s, l, m)$ is the normalisation matrix of the projection. In these matrices the bare potential is now buried. These are defined implicitly via the relation,

$$
\sum_{l, m}\left\langle\rho_{-\mathbf{p}}(t) \rho_{-\mathbf{s}}(t) \rho_{\mathbf{l}}(t) \rho_{\mathbf{m}}(t)\right\rangle g\left(l, m, s^{\prime}, p^{\prime}\right)=\delta_{\mathbf{s}, \mathbf{s}^{\prime}} \delta_{\mathbf{p}, \mathbf{p}^{\prime}}
$$

and it is clear from this that to explicitly evaluate $g(p, s, l, m)$ there is the need to evaluate four densities correlation functions, and this can be done only by introducing some approximation for the stochastic process. For the sake of simplicity, in the following we neglect $\Delta_{\mathbf{k}}(t)$ but later we will show how it can easily be included in the derivation, and how it might be important to do so.

The first important observation to make is that we can also think of performing the same projection onto density pairs as in (60) for the representation I of the random force (36). When this is done, it is easy to show that, at this level, this can be reproduced exactly, by making use of the definition of the normalisation matrix (61), without the need to calculate it explicitly. Let us show this in detail.

We want to calculate the coefficients of the projection for representation I of the reduced random force. We have,

$$
\begin{aligned}
& \left\langle\rho_{-\mathbf{l}}(t) \rho_{-\mathbf{m}}(t) \tilde{\eta}_{\mathbf{k}}^{(I)}(t)\right\rangle=-\frac{k^{2}}{\beta m} n c_{k}\left\langle\rho_{-\mathbf{l}}(t) \rho_{-\mathbf{m}}(t) \rho_{\mathbf{k}}(t)\right\rangle \\
& -\frac{1}{m V} \sum_{\mathbf{k}^{\prime}}\left(\mathbf{k} \cdot \mathbf{k}^{\prime}\right) v_{k^{\prime}}\left\langle\rho_{-\mathbf{l}}(t) \rho_{-\mathbf{m}}(t) \rho_{\mathbf{k}-\mathbf{k}^{\prime}}(t) \rho_{\mathbf{k}^{\prime}}(t)\right\rangle
\end{aligned}
$$

We note that these are static quantities, and thus the kinetic term in (36) can be exactly rewritten in terms of density when performing the averages, by separating out the velocity contribution. Thus, we insert these coefficients in the projection definition 
(60), and we have, omitting the time dependence since everything is at the same time $t$,

$$
\begin{aligned}
\tilde{\eta}_{\mathbf{k}}^{(I)} & =\sum_{l, m, p, s}\left\langle\rho_{-\mathbf{l}} \rho_{-\mathbf{m}} \tilde{\eta}_{\mathbf{k}}^{(I)}\right\rangle g(p, s, l, m) \rho_{\mathbf{p}} \rho_{\mathbf{s}}=-\frac{k^{2}}{\beta m} n c_{k} \sum_{p, s}\left\{\sum_{l, m}\left\langle\rho_{-\mathbf{l}} \rho_{-\mathbf{m}} \rho_{\mathbf{k}}\right\rangle g(p, s, l, m)\right\} \rho_{\mathbf{p}} \rho_{\mathbf{s}} \\
& -\frac{1}{m V} \sum_{\mathbf{k}^{\prime}}\left(\mathbf{k} \cdot \mathbf{k}^{\prime}\right) v_{k^{\prime}} \sum_{p, s}\left\{\sum_{l, m}\left\langle\rho_{-\mathbf{l}} \rho_{-\mathbf{m}} \rho_{\mathbf{k}-\mathbf{k}^{\prime}} \rho_{\mathbf{k}^{\prime}}\right\rangle g(p, s, l, m)\right\} \rho_{\mathbf{p}} \rho_{\mathbf{s}}
\end{aligned}
$$

We can thus evaluate explicitly the sums enclosed by graphs in (63) using (61) as,

$$
\begin{aligned}
& \left\{\sum_{l, m}\left\langle\rho_{-\mathbf{l}} \rho_{-\mathbf{m}} \rho_{\mathbf{k}}\right\rangle g(p, s, l, m)\right\}=\frac{1}{N} \delta_{\mathbf{p}, \mathbf{0}} \delta_{\mathbf{s}, \mathbf{k}} \\
& \left\{\sum_{l, m}\left\langle\rho_{-\mathbf{l}} \rho_{-\mathbf{m}} \rho_{\mathbf{k}-\mathbf{k}^{\prime}} \rho_{\mathbf{k}^{\prime}}\right\rangle g(p, s, l, m)\right\}=\delta_{\mathbf{p}, \mathbf{k}^{\prime}} \delta_{\mathbf{s}, \mathbf{k}-\mathbf{k}^{\prime}}
\end{aligned}
$$

and by inserting these results into (63), remembering that $\rho_{k=0} \equiv N$, we find,

$$
\tilde{\eta}_{\mathbf{k}}^{(I)}(t)=-\frac{k^{2}}{\beta m} n c_{k} \rho_{\mathbf{k}}(t)-\frac{1}{m V} \sum_{\mathbf{k}^{\prime}}\left(\mathbf{k} \cdot \mathbf{k}^{\prime}\right) v_{k^{\prime}} \rho_{\mathbf{k}-\mathbf{k}^{\prime}}(t) \rho_{\mathbf{k}^{\prime}}(t)
$$

which is exactly the original expression we had for $\tilde{\eta}_{\mathbf{k}}^{(I)}(t)$ before projecting it.

This result tells us that, since the two representations of the noise are intrinsically equal via the mapping between Newton and Langevin equations, also the projection of the reduced force in representation II onto density pairs in principle will be exact. Nonetheless, the problem with it lies in the fact that it cannot be calculated explicitly, because the definition (61) cannot be applied there to eliminate the unknown matrices $g$. Thus, differently from the original work on MCT [12], where the projection onto pairs was just chosen as the simplest approximation and not investigated further, we can now say, through our knowledge of the Newtonian representation which lies beneath oyr stochastic process, that it is an exact step, if taken carefully, and it does not need further terms, like for example one could think to project onto density triplets and so on, as a better form of approximation.

Nonetheless, this projection must be treated carefully. Indeed, it tells us that we have chosen the right subspace to project onto, but it also impose a further constraint on our system. As discussed previously, the equivalence between the two representations should, in principle, satisfy the constraints (39) at any order $n$. Here, since the use of representation II involves the projection onto density pairs, it is explicitly required that our system satisfies at least the second order type of constraint, expressed by (44).

Thus, this equation must be taken into account when using representation II of the random force to calculate the memory kernel, because the projection onto density pairs is now only exact when this constraint is satisfied.

\subsection{Calculation of the Memory Kernel for the Projected Representation II}

We now proceed to calculate the memory function using the projection onto density pairs of representation II. Concerning the integral term $\Delta_{\mathbf{k}}(t)$, we already know that 
its contribution is null when we use the Gaussian approximation to evaluate the exact formula for the memory kernel in representation I (45). In section 9, we will also reproduce the calculation of the kernel as in the original MCT derivation [12] and show that there the term $\Delta_{\mathbf{k}}(t)$ is simply neglected. Here, we will not neglect it and show that it is possible to incorporate it in the calculation, without rendering the calculation much harder. Thus, as illustrated in (60), we do not project it onto density pairs.

Using the explicit expression for the coefficients of the projection for the second derivative term, given in (42) and (43), we can write the 'exact' expression for the memory function, 'exact' because it is only to be considered exact when used in conjunction with the equation of constraint (44), as discussed in the previous paragraph. Thus, applying (23), and performing the sums that can be done explicitly so to isolate the various types of density correlators, we have,

$$
\begin{aligned}
& \gamma_{\mathbf{k}}^{(I I)}(t)=\frac{N}{\beta m k^{2}} \sum_{m, p, s} \sum_{m^{\prime}, p^{\prime} s^{\prime}}\left[k^{2}-\mathbf{k} \cdot \mathbf{m}^{\prime} n c_{m^{\prime}}-\mathbf{k} \cdot\left(\mathbf{k}-\mathbf{m}^{\prime}\right) n c_{k-m^{\prime}}\right]\left[k^{2}-\mathbf{k} \cdot \mathbf{m} n c_{m}-\right. \\
& \left.\mathbf{k} \cdot(\mathbf{k}-\mathbf{m}) n c_{k-m}\right] S_{m^{\prime}} S_{k-m^{\prime}} S_{m} S_{k-m} g(p, s, k-m, m) g\left(k-m^{\prime}, m^{\prime}, p^{\prime}, s^{\prime}\right)\left\langle\rho_{-\mathbf{p}^{\prime}}(0) \rho_{-\mathbf{s}^{\prime}}(0) \rho_{\mathbf{p}}(t) \rho_{\mathbf{s}}(t)\right\rangle \\
& -\frac{1}{\beta m S_{k}} \sum_{m, p, s}\left[k^{2}-\mathbf{k} \cdot \mathbf{m} n c_{m}-\mathbf{k} \cdot(\mathbf{k}-\mathbf{m}) n c_{k-m}\right] S_{m} S_{k-m} g(p, s, k-m, m)\left\langle\rho_{-\mathbf{k}}(0) \rho_{\mathbf{p}}(t) \rho_{\mathbf{s}}(t)\right\rangle \\
& -\frac{1}{\beta m S_{k}} \sum_{m^{\prime}, p^{\prime}, s^{\prime}}\left[k^{2}-\mathbf{k} \cdot \mathbf{m}^{\prime} n c_{m^{\prime}}-\mathbf{k} \cdot\left(\mathbf{k}-\mathbf{m}^{\prime}\right) n c_{k-m^{\prime}}\right] S_{m^{\prime}} S_{k-m^{\prime}} g\left(k-m^{\prime}, m^{\prime}, p^{\prime}, s^{\prime}\right) \\
& \left\langle\rho_{-\mathbf{p}^{\prime}}(0) \rho_{-\mathbf{s}^{\prime}}(0) \rho_{\mathbf{k}}(t)\right\rangle-\frac{1}{k^{2}} \sum_{m^{\prime}, p^{\prime}, s^{\prime}}\left[k^{2}-\mathbf{k} \cdot \mathbf{m}^{\prime} n c_{m^{\prime}}-\mathbf{k} \cdot\left(\mathbf{k}-\mathbf{m}^{\prime}\right) n c_{k-m^{\prime}}\right] S_{m^{\prime}} S_{k-m^{\prime}} g\left(k-m^{\prime}, m^{\prime}, p^{\prime}, s^{\prime}\right) \\
& \int_{0}^{t} d t^{\prime} \gamma_{\mathbf{k}}\left(t-t^{\prime}\right)\left\langle\rho_{-\mathbf{p}^{\prime}}(0) \rho_{-\mathbf{s}^{\prime}}(0) \dot{\rho}_{\mathbf{k}}\left(t^{\prime}\right)\right\rangle+\frac{k^{2}}{\beta m S_{k}^{2}} S_{k}(t)+\frac{1}{N S_{k}} \int_{0}^{t} d t^{\prime} \gamma_{\mathbf{k}}\left(t-t^{\prime}\right)\left\langle\rho_{-\mathbf{k}}(0) \dot{\rho}_{\mathbf{k}}\left(t^{\prime}\right)\right\rangle
\end{aligned}
$$

This form has now the same structure as expression (45) for representation I. In other words, it is explicitly visible that it contains the time-dependent 4-point correlation $\left\langle\rho_{-\mathbf{p}^{\prime}}(0) \rho_{-\mathbf{s}^{\prime}}(0) \rho_{\mathbf{p}}(t) \rho_{\mathbf{s}}(t)\right\rangle$, as well as triplets and pairs time-dependent correlations. Nonetheless, now the coupling coefficients do not contain the bare interactions, but already we can see that they are composed of static quantities, such as the direct correlation function, although clearly the unknown matrix $g$ still needs to be treated.

\subsection{The Gaussian case}

In this section we want to apply the simple Gaussian approximation for the random process that we used to calculate the memory kernel in representation I, to the case of projected representation II. The first equation to study is of course (44), because this is the one that guarantees the consistency of the whole scheme. Thus, recalling that for a gaussian noise multiple averages can be decomposed as in (48), the constraint (44) becomes in this simple case,

$$
\begin{aligned}
& -\frac{1}{V}\left\{\mathbf{k} \cdot \mathbf{p} v_{p}+\mathbf{k} \cdot(\mathbf{k}-\mathbf{p}) v_{|\mathbf{k}-\mathbf{p}|}\right\} N^{2} S_{p} S_{|\mathbf{k}-\mathbf{p}|}-\frac{k^{2}}{\beta}\left\{\delta_{\mathbf{p}, 0}+\delta_{\mathbf{p}, \mathbf{k}}\right\} N^{2} S_{k}= \\
& -\frac{N}{\beta}\left\{k^{2}-\mathbf{k} \cdot \mathbf{p} n c_{p}-\mathbf{k} \cdot(\mathbf{k}-\mathbf{p}) n c_{|\mathbf{k}-\mathbf{p}|}\right\} S_{p} S_{|\mathbf{k}-\mathbf{p}|}
\end{aligned}
$$


Now using the condition $c_{k}=-\beta v_{k}$, that we found applying the gaussian approximation to the YBG equation, we have cancellation of some terms, but then this would imply, $\left\{\delta_{\mathbf{p}, 0}+\delta_{\mathbf{p}, \mathbf{k}}\right\} N S_{k}=S_{p} S_{|\mathbf{k}-\mathbf{p}|}$ and this is clearly not an equality valid for all $\mathbf{k}$ and $\mathbf{p}$, but only for the special cases $\mathbf{k}=\mathbf{p}$, or $\mathbf{p}=0$. Thus, we conclude that equation (44) cannot be satisfied by a simple gaussian approximation. This, indeed, implies that using representation II, projected onto density pairs, and proceeding with a pure gaussian approximation to calculate the memory kernel, the result found for it will not be the same as the one found with representation I (56), because the relation of constraint (44) would be violated in this way. As a consequence of this, we have that, to use representation II, one must go a step further in the approximation of the noise than with representation I, where the simple gaussianity of the noise is sufficient to develop a consistent theory. This comes from the fact that representation I is intrinsically exact, while representation II is not, with respect to the projection onto pairs, and thus, using the latter, one must be more careful.

To evaluate explicitly (67), we need an expression for the normalisation matrix $g$. Using again gaussian properties of the noise to decompose density correlations, we have that (61) gives,

$$
g\left(s, p, p^{\prime}, s^{\prime}\right) \approx \frac{\delta_{\mathbf{p}, \mathbf{p}^{\prime}} \delta_{\mathbf{s}, \mathbf{s}^{\prime}}}{2 N^{2} S_{s} S_{p}}
$$

Using this result, and applying systematically gaussian decomposition of averages at equal and unequal times, we have the following result for the memory function,

$$
\begin{aligned}
\gamma_{\mathbf{k}}^{(I I) \text { Gauss }}(t)= & \frac{1}{2 \beta m k^{2} N} \sum_{p}\left[k^{2}-\mathbf{k} \cdot \mathbf{p} n c_{p}-\mathbf{k} \cdot(\mathbf{k}-\mathbf{p}) n c_{|\mathbf{k}-\mathbf{p}|}\right]^{2} S_{p}(t) S_{|\mathbf{k}-\mathbf{p}|}(t) \\
& \frac{k^{2}}{\beta m S_{k}}\left(1-n c_{k}\right) S_{k}(t)-\left[\left(1-n c_{k}\right)-\frac{1}{S_{k}}\right] \int_{0}^{t} \gamma_{\mathbf{k}}\left(t-t^{\prime}\right) \dot{S}_{k}\left(t^{\prime}\right)
\end{aligned}
$$

The first thing to note here is that the contribution, given by the integral term, vanishes because $\left[\left(1-n c_{k}\right)-1 / S_{k}\right]=0$, and this coincides with what we have found in representation I in gaussian approximation. Also, we can isolate from the sum the terms for which $m$ is singular $(m=0$ and $m=k$ ), and, recalling equation (57), we can rewrite $(70)$ as,

$$
\gamma_{\mathbf{k}}(t)=\gamma_{\mathbf{k}}(t) \stackrel{M C T}{+} \frac{1}{2 \beta m k^{2} N} \sum_{\mathbf{p} \neq 0, \mathbf{k}}\left[k^{4}-2 \mathbf{k} \cdot \mathbf{p} n c_{p}-2 \mathbf{k} \cdot(\mathbf{k}-\mathbf{p}) n c_{|\mathbf{k}-\mathbf{p}|}\right] S_{p}(t) S_{|\mathbf{k}-\mathbf{p}|}(t)
$$

Thus, clearly, this way of proceeding does not reproduce MCT result and the reason of this lies in the fact that the two representations for the noise (36), (37) are not equivalent under projection onto density pairs using a simple Gaussian approximation, because, as shown above (44) is not satisfied, except for the trivial wave-vectors cases. This means that further investigation of the theory must involve a new type of approximation for multiple density averages, that would satisfy firstly equation (44). 


\subsection{The STLS Approximation: a step beyond Gaussianity}

We want to illustrate here a step in the direction of improving the gaussian approximation for density averages. Indeed, turning to consider other known approximations in literature, it may be possible to satisfy the constraint (44) for a larger number of wave-vectors than the simple gaussian one. The example we will refer to is known as the Singwi-Tosi-Land-Sjolander approximation (STLS) [19]. What we show here does not lead to a definitive result for corrections to MCT, but it is illustrative of the issues involved.

We can consider now for example the triple average in the YBG equation (19). Recalling what happened with the Gaussian approximation, we can now think that in this new scheme, we will have, as dominant contributions to the sum, not only the Gaussian terms but also some corrections to them, that would be most meaningful if they in some way include the gaussian terms, so that the new scheme is actually an improvement of the old one. To say this mathematically, we can write explicitly,

$$
\begin{aligned}
& \left\langle\rho_{-\mathbf{k}}(t) \rho_{\mathbf{k}-\mathbf{k}^{\prime}}(t) \rho_{\mathbf{k}^{\prime}}(t)\right\rangle=N\left\{\delta_{\mathbf{k}, 0}+\mathcal{C}_{1}\right\}\left[S_{k^{\prime}}+N \delta_{\mathbf{k}^{\prime}, 0}\right] \\
& +N\left\{\delta_{\mathbf{k}, \mathbf{k}^{\prime}}+\mathcal{C}_{2}\right\}\left[S_{k}+N \delta_{\mathbf{k}, 0}\right]+N\left\{\delta_{\mathbf{k}^{\prime}, 0}+\mathcal{C}_{3}\right\}\left[S_{k}+N \delta_{\mathbf{k}, 0}\right]
\end{aligned}
$$

where $\mathcal{C}_{1,2,3}$ are the three corrections to the Gaussian terms, that are simply $\delta$-functions. Thus, we are still assuming that the triplet correlation function decomposes into three separate contributions, as for the gaussian variables, but now these contributions will be more complicated. Following the literature [19], the STLS consists in assuming the following,

$$
\begin{aligned}
& \rho_{\mathbf{k}-\mathbf{k}^{\prime}}(t) \rho_{\mathbf{k}^{\prime}}(t)=\sum_{n} e^{i\left(\mathbf{k}-\mathbf{k}^{\prime}\right) \cdot \mathbf{r}_{n}(t)} \sum_{n^{\prime}} e^{i \mathbf{k}^{\prime} \cdot \mathbf{r}_{n^{\prime}}(t)}=\sum_{n^{\prime}} e^{i \mathbf{k} \cdot \mathbf{r}_{n^{\prime}}(t)} \sum_{n} e^{i\left(\mathbf{k}-\mathbf{k}^{\prime}\right) \cdot\left(\mathbf{r}_{n}(t)-\mathbf{r}_{n^{\prime}}(t)\right)} \\
& \approx \sum_{n^{\prime}} e^{i \mathbf{k} \cdot \mathbf{r}_{n^{\prime}}(t)}\left\langle\sum_{n} e^{i\left(\mathbf{k}-\mathbf{k}^{\prime}\right) \cdot\left(\mathbf{r}_{n}(t)-\mathbf{r}_{n^{\prime}}(t)\right)}\right\rangle=\rho_{\mathbf{k}}(t)\left[S_{\left|\mathbf{k}-\mathbf{k}^{\prime}\right|}+N \delta_{\mathbf{k}, \mathbf{k}^{\prime}}\right]
\end{aligned}
$$

where the approximation made consists of replacing the sum over $n$ on the second line of (73) by its average, as it was independent on $n^{\prime}$.

If we apply this to the triplet correlation function above, we have,

$$
\begin{aligned}
& \left\langle\rho_{-\mathbf{k}}(t) \rho_{\mathbf{k}-\mathbf{k}^{\prime}}(t) \rho_{\mathbf{k}^{\prime}}(t)\right\rangle=N\left\{\delta_{\mathbf{k}, 0}+S_{k}\right\}\left[S_{k^{\prime}}+N \delta_{\mathbf{k}^{\prime}, 0}\right]+ \\
& +N\left\{\delta_{\mathbf{k}, \mathbf{k}^{\prime}}+S_{\left|\mathbf{k}-\mathbf{k}^{\prime}\right|}\right\}\left[S_{k}+N \delta_{\mathbf{k}, 0}\right]+N\left\{\delta_{\mathbf{k}^{\prime}, 0}+S_{k^{\prime}}\right\}\left[S_{k}+N \delta_{\mathbf{k}, 0}\right]
\end{aligned}
$$

and the corrections $\mathcal{C}_{1,2,3}$ are simply the static structure factors, with wavenumber corresponding to the respective $\delta$-function. A physical picture of what this represents is straightforward. Indeed, the gaussian decomposition of averages simply means that the densities at three points are only coupled two by two in all possible combinations, assuming that the third one is infinitely far from the others and thus, this is averaged out. The new STLS approach consists in accounting for some aspects of the correlations of

the third particle. This is the reason why we now need two structure factors to describe our triplet correlations, while in the gaussian treatment we needed only one. 
Now we apply the approximation (74) to the YBG equation (19), thus obtaining a new solution for the direct correlation function of the system, i.e.

$$
c_{k}=-\beta v_{k}-\frac{\beta}{N k^{2}} \sum_{\mathbf{k}^{\prime} \neq \mathbf{k}} v_{k^{\prime}}\left(\mathbf{k} \cdot \mathbf{k}^{\prime}\right) S\left(\left|\mathbf{k}-\mathbf{k}^{\prime}\right|\right)
$$

where again only the contribution driven by the $\mathbf{k}=\mathbf{k}^{\prime} \delta$-function survives, because the other two can be both shown to have spherical symmetry and thus, their integral is null. The result (75) clearly still contains the information of the RPA in the first term, but adds to it a new term to be taken into account for the calculation of $S(k)$ from the potential.

The next step now is to see what happens when applying the STLS approximation to the second equation of constraint (44). We have seen that the Gaussian approximation would satisfy this condition only for two singular wavevectors. Using the STLS approximation for the quadruplet and triplet correlations on the left hand side and (75) for the direct correlation function on the right hand side, (44) becomes

$$
\begin{aligned}
& \sum_{\mathbf{k}^{\prime}} v_{k^{\prime}}\left(\mathbf{k} \cdot \mathbf{k}^{\prime}\right)\left[S_{k} S_{p} S_{k^{\prime}}+\left(S_{\left|\mathbf{k}^{\prime}-\mathbf{p}\right|}+S_{\left|\mathbf{k}-\mathbf{k}^{\prime}-\mathbf{p}\right|}\right) S_{p} S_{|\mathbf{k}-\mathbf{p}|}\right]= \\
& \sum_{\mathbf{k}^{\prime}} v_{k^{\prime}}\left[\frac{(\mathbf{k} \cdot \mathbf{p})\left(\mathbf{k}^{\prime} \cdot \mathbf{p}\right)}{p^{2}} S_{\left|\mathbf{k}^{\prime}-\mathbf{p}\right|}+\frac{(\mathbf{k} \cdot(\mathbf{k}-\mathbf{p}))\left(\mathbf{k}^{\prime} \cdot(\mathbf{k}-\mathbf{p})\right)}{(|\mathbf{k}-\mathbf{p}|)^{2}} S_{\left|\mathbf{k}-\mathbf{k}^{\prime}-\mathbf{p}\right|}\right] S_{p} S_{|\mathbf{k}-\mathbf{p}|}
\end{aligned}
$$

The first of the three terms on the left hand side gives no contribution due to spherical symmetry. Thus, to satisfy the constraint (44) in the STLS approximation, we have to satisfy the general condition for certain wave-vectors $\mathbf{p}$, equivalently $\mathbf{k}-\mathbf{p}$,

$$
\left(\mathbf{k} \cdot \mathbf{k}^{\prime}\right)=\frac{(\mathbf{k} \cdot \mathbf{p})\left(\mathbf{k}^{\prime} \cdot \mathbf{p}\right)}{p^{2}}
$$

This implies the geometrical condition $\cos \theta_{k, k^{\prime}}=\cos \theta_{k, p} \cos \theta_{k^{\prime}, p}$, which is equivalent to $\sin \theta_{k, p} \sin \theta_{k^{\prime}, p} \cos \phi_{k, k^{\prime}}=0$. Being here $\mathbf{k}^{\prime}$ arbitrary, the only general condition that can satisfy this constraint is that $\mathbf{k}$ and $\mathbf{p}$ are parallel, plus of course of both of them being zero. This is certainly an enlarged number of wave-vectors over which STLS satisfies the second order constraint (44) with respect to the only two suitable values for the gaussian approximation. This type of scheme would possibly provide a better way of proceeding in calculation of the memory kernel, using the projection onto density pairs of representation II. A problem, in doing so, would be how to extend correctly the STLS approximation $(\sqrt{73})$ to unequal time correlations, and if then would then be possible to close the theory, by eliminating the bare potential, for example using the result of the YBG $(75)$.

These questions do not appear to have an easy answer, as well as the challenge of satisfying the constraint (44) for any wave-vector seems quite hard. That is why future work will be addressed to propose an alternative, in principle self-consistently determined, way to go beyond the gaussian closure, as well as to renormalize the bare interactions. 


\section{Comparison of our formalism with the original derivation of MCT}

Here, we want to link our formalism the traditional derivation of MCT [12], based on the projector operator formalism. In this way, we will be able to show in more detail the various steps that were originally taken, and compare them to our work.

Thus, we analyze the original derivation, starting form when equations of motion are written for the density and current correlators, following the Zwanzig-Mori (ZM) formalism, where the variables which define the projection space are, indeed, the density $\rho_{\mathbf{k}}$ and the longitudinal current $\mathbf{J}_{\mathbf{k}}$ [12]. In the subspace spanned by these variables, the projection operators are defined as $\mathcal{P}$ being the projector onto the subspace itself and $\mathcal{Q}=1-\mathcal{P}$ the orthogonal projector. Thus, translating the notation in [12] into our notation, we have the following equations of motion,

$$
\left(\begin{array}{c}
\dot{\rho}_{\mathbf{k}}(t) \\
\mathbf{j}_{\mathbf{k}}(t)
\end{array}\right)-i\left(\begin{array}{cc}
0 & k \\
\Omega_{k} / k & 0
\end{array}\right)\left(\begin{array}{c}
\rho_{\mathbf{k}}(t) \\
\mathrm{J}_{\mathbf{k}}(t)
\end{array}\right)+\int_{0}^{t}\left(\begin{array}{cc}
0 & 0 \\
0 & \gamma_{\mathbf{k}}\left(t-t^{\prime}\right)
\end{array}\right)\left(\begin{array}{c}
\rho_{\mathbf{k}}\left(t^{\prime}\right) \\
\mathbf{J}_{\mathbf{k}}\left(t^{\prime}\right)
\end{array}\right)=\left(\begin{array}{c}
0 \\
R_{\mathbf{k}}(t)
\end{array}\right)(78)
$$

that combined together give equation (21), where $R_{\mathbf{k}}(t)$ is the random force which was indicated as $\eta_{\mathbf{k}}(t)$ in our notation. There are a few things to specify regarding (78). Firstly, in the ZM formalism, the random force at the initial time $t=0$, is defined for a general variable $\mathcal{A}$ as $R_{\mathcal{A}(0)}=\mathcal{Q} \dot{\mathcal{A}}$, and so it is clear that $\mathcal{Q} \dot{\rho}_{\mathbf{k}}=0$ and the only non-vanishing random force is related to the current. Thus we have,

$$
R_{\mathbf{k}}(0)=\mathcal{Q} \mathbf{j}_{\mathbf{k}}(0)=-i / k\left[\ddot{\rho}_{\mathbf{k}}(0)+\Omega_{k} \rho_{\mathbf{k}}(0)\right]
$$

Also, in this formalism, we know that the random force evolves in time not with the Liouvillian operator $\mathcal{L}$, but with a particular projection of it, and thus,

$$
R_{\mathbf{k}}(t)=\exp (i \mathcal{Q} \mathcal{L} \mathcal{Q} t) R_{\mathbf{k}}(0) .
$$

Nonetheless, in the MCT approach, the evolution of the random force is approximated with the Liouvillian evolution in time, i.e. with $e^{i \mathcal{L} t} R_{\mathbf{k}}(0)$. Thus, the approximation corresponds to neglect the difference between $e^{i \mathcal{Q} \mathcal{L Q} t} R_{\mathbf{k}}(0)$ and $e^{i \mathcal{L} t} R_{\mathbf{k}}(0)$ i.e.

$$
R_{\mathbf{k}}(t) \approx-\frac{i}{k}\left[\ddot{\rho}_{\mathbf{k}}(t)+\Omega_{k} \rho_{\mathbf{k}}(t)\right] .
$$

In our derivation, using representation II, the true random force $\eta_{\mathbf{k}}(t)$ is given in (37), and it is clear that, apart from the factor $-i / k$ that comes from using as a variable $\mathbf{j}_{\mathbf{k}}(t)$ instead of $\dot{\rho}_{\mathbf{k}}(t)$, the two being related via the relation $\dot{\rho}_{\mathbf{k}}(t)=i k \mathrm{j}_{\mathbf{k}}(t)$, this is the same as the exact $R_{\mathbf{k}}(t)$ (80). The comparison between (37) and (81) allows us to give an exact expression for the difference between the true random force and the MCT approximated one. Indeed, it turns out to be the integral term $\Delta_{\mathbf{k}}(t)$, defined in (38). We have shown how to include $\Delta_{\mathbf{k}}(t)$ in the equations for the memory kernel. We have also shown that the contribution to the memory kernel coming from $\Delta_{\mathbf{k}}(t)$ is strictly zero in RPA, in both representations.

Thus, the starting point for the original derivation of the memory kernel in MCT, is equation (67), in which one neglects the integral term. The approximations that are then made are the following. Firstly, it is chosen to perform a kind of a gaussian 
approximation for the four-point density average, which means that still the quadruple averages factorises into averages of pairs, but not all the possible combinations are considered, i.e.

$$
\left\langle\rho_{-\mathbf{p}^{\prime}}(0) \rho_{-\mathbf{s}^{\prime}}(0) \rho_{\mathbf{p}}(t) \rho_{\mathbf{s}}(t)\right\rangle \approx\left\langle\rho_{-\mathbf{p}^{\prime}}(0) \rho_{\mathbf{p}}(t)\right\rangle\left\langle\rho_{-\mathbf{s}^{\prime}}(0) \rho_{\mathbf{s}}(t)\right\rangle N^{2} S_{p}(t) S_{s}(t) \delta_{\mathbf{p}, \mathbf{p}^{\prime}} \delta_{\mathbf{s}, \mathbf{s}^{\prime}}
$$

The same approach is followed to evaluate the normalization matrix $g$, using definition (61), i.e. $g(l, m, p, s) \approx \delta_{\mathbf{l}, \mathbf{s}} \delta_{\mathbf{m}, \mathbf{p}} / N^{2} S_{p} S_{s}$. These types of simplified gaussian approximations can be substituted by the conventional ones that we have used, like for example (53), without changing the final result for the MCT memory kernel (57), because of the symmetry that this has with respect to the wave-vectors $\mathbf{p}$ and $\mathbf{q}$. Regarding, instead, the triple averages appearing in the coefficients, they can be exactly written as, for example,

$$
\left\langle\rho_{-\mathbf{l}}(t) \rho_{-\mathbf{m}}(t) \rho_{\mathbf{k}}(t)\right\rangle=N \delta_{\mathbf{l}+\mathbf{m}, \mathbf{k}} S_{l} S_{m} S_{k}\left(1+n c_{3}\right)
$$

where $c_{3}$ indicates the direct correlation function for triplets. Thus, based on the observation that for hard spheres systems it has been shown that the contribution given by $c_{3}$ in (83) is very small, this is neglected. Also, if one wanted to include them in the calculation, they can be easily calculated from simulations. Thus, this approximations for the triplet density correlations appears to be more advanced than the simple decomposition of it, generated by a gaussian noise choice, as in (49). Nonetheless, this breaks the full consistency of the scheme of approximations in developing the theory.

Eq. (83), but where the triplet direct correlation function is neglected, leads to a crucial simplification of the coefficients of the projection of the noise. Indeed, it implies a cancellation of the triplet term with the term in $k^{2}$. Thus, one can see that this, in addition to the gaussian type of approximation for the quadruple correlations and the normalisation matrix discussed above, leads very easily to the well known expression for $\gamma_{\mathbf{k}}^{M C T}(t)$ given in (57).

Now let us analyze what the inclusion of the integral term $\Delta_{\mathbf{k}}(t)$ would produce in the present scheme. To calculate the full memory kernel we are left to evaluate the last term in (67). Using the approximations just discussed, i.e. gaussian averages for 4-point correlations and (83) for the triplets neglecting $c_{3}$, we have that the last term reduces to,

$$
\begin{aligned}
& \frac{\beta m}{N k^{2}} \sum_{l^{\prime}, m^{\prime}, p^{\prime}, s^{\prime}}\left\{-\frac{N}{\beta m}\left[k^{2}-\mathbf{k} \cdot \mathbf{m}^{\prime} n c_{m^{\prime}}-\mathbf{k} \cdot\left(\mathbf{k}-\mathbf{m}^{\prime}\right) n c_{k-m^{\prime}}\right] S_{m^{\prime}} S_{q-m^{\prime}} \delta_{l^{\prime}+m^{\prime}, k}\right. \\
& \left.+\frac{k^{2}}{\beta m S_{k}}\left\langle\rho_{-\mathbf{k}}(0) \rho_{\mathbf{l}^{\prime}}(0) \rho_{\mathbf{m}^{\prime}}(0)\right\rangle\right\} g\left(l^{\prime}, m^{\prime}, p^{\prime}, s^{\prime}\right) \int_{0}^{t} d t^{\prime} \gamma_{\mathbf{k}}\left(t-t^{\prime}\right)\left\langle\rho_{-\mathbf{p}^{\prime}}(0) \rho_{-\mathbf{s}^{\prime}}(0) \dot{\rho}_{\mathbf{k}}\left(t^{\prime}\right)\right\rangle \\
& =\frac{\beta m}{N^{3} k^{2}} \sum_{m^{\prime}}\left[\mathbf{k} \cdot \mathbf{m}^{\prime} n c_{m^{\prime}}+\mathbf{k} \cdot\left(\mathbf{k}-\mathbf{m}^{\prime}\right) n c_{k-m^{\prime}}\right] \int_{0}^{t} d t^{\prime} \gamma_{\mathbf{k}}\left(t-t^{\prime}\right)\left\langle\rho_{-\mathbf{m}^{\prime}}(0) \rho_{-\mathbf{k}+\mathbf{m}^{\prime}}(0) \dot{\rho}_{\mathbf{k}}\left(t^{\prime}\right)\right\rangle
\end{aligned}
$$

Thus, there is the need to calculate the dynamical average $\left\langle\rho_{-\mathbf{m}^{\prime}}(0) \rho_{-\left(\mathbf{k m}^{\prime}\right)}(0) \dot{\rho}_{\mathbf{k}}\left(t^{\prime}\right)\right\rangle$ to evaluate the contribution of this term. We could use either the Gaussian approximation, as for the quadruple average (82), or a generalization for unequal times of the triplet expression (83). Nonetheless, in both cases, this contribution does not appear to be zero, 
as in purely gaussian treatments, and moreover, it requires the introduction of another approximation, somewhat consistent with the others that have been made previously. In any case, it would be certainly different from zero, and its simple neglection, without a consistent justification of it, is clearly unsatisfying.

\section{Conclusions}

In this paper we have attempted to provide an alternative way to derive a mode-coupling theory. We have shown how Newton's equations can be represented by trial equations, and how these may be approximated. The approach starting from Newton's equations allows us to derive explicit and exact expressions for the the memory function and for the noise without the requirement of projection operators.It thereby provides an alternative view that may lead to new insights. The resulting equations, solved under the assumption that the noise, and consequently the density fluctuations of the liquid, is gaussian distributed, are equivalent to the random-phase-approximation for the static structure factor and to the well known ideal mode coupling theory (MCT) equations for the dynamics. This suggests that MCT is a theory of fluid dynamics that becomes exact in a mean-field limit. This possibility was suggested some time ago on the basis of the analogies between the equations describing the schematic MCT models and the the dynamics of the order parameter in disordered $p$-spin models, solved under strict mean-field approximation [14, 15, 24].

We have also investigated in detail the basic meaning of the less controlled steps intrinsic in the derivation of conventional MCT [12]. One important point is the demonstration that, if the projection of the noise over the density pairs could be carried out without approximations, the resulting expression would be exact. The evaluation of the quadruplet correlation functions under gaussian statistics, an approximation employed in the evaluation of the memory function when projected in the density pair subspace constitutes, in conventional MCT, the step equivalent to the RPA approximation. Hence, our approach suggests that the conventional MCT is exact in the RPA limit. This offers the possibility of improving the theory by improving the approximation of the four-point correlation functions in the same way as one improves the static structure factors on moving beyond RPA. We have illustrated an example of this by using the STLS approximation (73), which leads to a different closure for the YBG equation (75), certainly more advanced than the simple RPA.

The implication of this is clearly not that ideal MCT is a theory which can be applied only in the weak-coupling limit, where RPA becomes exact. In fact, the RPA limit that we have studied may in principle be corrected in two generic manners. Firstly, the treatment of the static correlations may be improved by use of a better direct correlation function than that implied by the consistent theory. On the other hand,

Kawasaki 25] has presented an interesting derivation of MCT based on the (quadratic) density functional Ramakrishnan-Yussouf free energy of a liquid, where the effective interaction between the density pairs is exactly $-c(k) / \beta$, thereby accomplishing the 
limited form of renormalisation implied by RPA. In addressing this issue we note that the comparison between the MCT prediction and hard-core type experimental [26, 27] or numerical [28] results in dense liquid states are sometimes astonishingly good, even for network forming liquids 29, 30].

However, the presence of the bare interactions in our formulation of the theory constitutes a strong limitation to our approach, and, to our present knowledge, the gaussian approximation, both for static and dynamics, appears to be the only immediate

fully consistent closure. Thus, the main problem in developing further this formalism to better types of approximations is how to renormalise more effectively the interactions, othen than simply RPA. We will attempt to do so in future work.

\section{References}

[1] L. Boltzmann, Lectures on Gas Theory, University California Press, Berkeley (1964).

[2] R. Zwanzig, in Lectures of Theoretical Physics, Vol. III, Wiley Interscience, New York (1961); H. Mori, Prog. Theor. Phys. 33, 423; 34, 399 (1965).

[3] J. P. Hansen and I. R. McDonald, Theory of simple liquids, Academic Press, London (1986), 2nd Edition.

[4] P. W. Anderson, Basic notions of condensed matter physics, Benjamin-Cummings, London (1984).

[5] For review see M. Mezard, G. Parisi and M. A. Virasoro, Spin Glass Theory and Beyond, in World Scientific Lecture Notes in Physics, Vol. 9, World Scientific, Singapore (1987).

[6] For recent review see articles in Science 267, (1995) and M.D. Ediger, C.A. Angell and S.R. Nagel, J. Phys. Chem 100, 13200 (1996).

[7] For a recent review see P.G. Debenedetti and F. H. Stillinger, Nature, 410, 259 (2001).

[8] J. Bergenholtz and M. Fuchs, Phys. Rev. E 59, 5706 (1999).

[9] K.A. Dawson, G. Foffi, M.Fuchs, W.Götze, F. Sciortino, M. Sperl, P. Tartaglia, Th. Voigtmann and E. Zaccarelli, Phys. Rev. E 63,11401 (2001).

[10] E. Zaccarelli, G. Foffi, K.A. Dawson, F. Sciortino, and P. Tartaglia, Phys. Rev. E 63, 31501 (2001).

[11] U. Bengtzelius, W. Götze and A. Sjölander, J. Phys. C 17, 5915 (1984).

[12] W. Götze, in Liquids, Freezing and Glass Transition, edited by J.P. Hansen, D.Levesque and J.Zinn-Justin (North-Holland, Amsterdam, 1991), p.287.

[13] E. Zaccarelli, G. Foffi, F. Sciortino, P. Tartaglia and K. A. Dawson, Europhysics Letts, 55, 139 (2001).

[14] T. R. Kirkpatrick and D. Thirumalai, Phys. Rev. Lett. 58, 2091 (1987).

[15] A. Crisanti and H.-J. Sommers, Z. Physik B 87, 341 (1992).

[16] J. K. Percus, in Studies in Statistical Mechanics, Vol. VIII, edited by E. W. Montroll and J.L. Lebowitz (North-Holland, Amsterdam, 1982).

[17] A. N.H.March and M.P.Tosi, Atomic dynamics in liquids, Dover (NY) 1991.

[18] R. Kubo, M. Toda and N. Hashitsume, Statistical Physics II, Springer-Verlag, Berlin (1991), 2nd Edition.

[19] K. S. Singwi, M.P. Tosi, R.H. Land, A. Sjölander, Phys. Rev. 176, 589 (1968).

[20] L. D. Landau and E. M. Lifshitz, Fluid Mechanics, Pergamon, Oxford (1987), 2nd edition.

[21] R. Zwanzig, Phys. Rev. 156, 190 (1967).

[22] T. Keyes and I. Oppenheim, Phys. Rev. A 8, 937-949 (1973).

[23] P. De Gregorio, F. Sciortino, P. Tartaglia, E. Zaccarelli and K. A. Dawson, Physica A (in press), 2001.

[24] J-P. Bouchad, L. Cugliandolo, J. Kurchan and M. Mezard, Physica A 226, 243 (1996).

[25] K. Kawasaki, Transp. Theory Stat. Phys. 24, 755 (1995); Physica A 208, 35 (1994).

[26] W. Götze, J. Phys.: Condens. Matter 11, A1 (1999). 
[27] W. van Megen, S. M. Underwood and P. N. Pusey, Phys. Rev. Lett. 67, 1586 (1991);W. van Megen and S. M. Underwood, Phys. Rev. Lett. 70, 2766 (1993); W. van Megen and S. M. Underwood, Phys. Rev. Lett. 72, 1773 (1994).

[28] M. Nauroth and W. Kob, Phys. Rev. E 55, 675 (1997). T. Gleim, W. Kob, K. Binder, Phys. Rev. Lett. 81, 4404 (1998).

[29] L. Fabbian, A. Latz, R. Schilling, F. Sciortino, P. Tartaglia and C. Theis, Phys. Rev. E. 605768 (1999).

[30] F. Sciortino and W. Kob, Phys. Rev. Lett. 86,648 (2001). 